\title{
BLAST OBSERVATIONS OF RESOLVED GALAXIES: TEMPERATURE PROFILES AND THE EFFECT OF ACTIVE GALACTIC NUCLEI ON FIR TO SUBMILLIMETER EMISSION
}

\author{
Donald V. Wiebe ${ }^{1,2}$, Peter A. R. Ade ${ }^{3}$, James J. Bock ${ }^{4,5}$, Edward L. Chapin ${ }^{1}$, Mark J. Devlin ${ }^{6}$, Simon Dicker $^{6}$, \\ Matthew Griffin ${ }^{3}$, Joshua O. Gundersen ${ }^{7}$, Mark Halpern ${ }^{1}$, Peter C. Hargrave ${ }^{3}$, David H. Hughes ${ }^{8}$, Jeff Klein ${ }^{6}$, \\ Gaelen Marsden $^{1}$, Peter G. Martin ${ }^{9}, 10$, Philip Mauskopf ${ }^{3}$, Calvin B. Netterfield $^{2,10}$, Luca Olmi $^{11,12}$, Enzo Pascale $^{3}$, \\ Guillaume Patanchon ${ }^{13}$, Marie Rex ${ }^{6}$, Douglas ScotT $^{1}$, Christopher Semisch $^{6}$, Nicholas Thomas ${ }^{7}$, \\ Matthew D. P. Truch ${ }^{6}$, Carole Tucker $^{3}$, Gregory S. Tucker ${ }^{14}$, and Marco P. Viero ${ }^{10}$ \\ ${ }^{1}$ Department of Physics \& Astronomy, University of British Columbia, 6224 Agricultural Road, Vancouver, BC V6T 1Z1, Canada \\ ${ }^{2}$ Department of Physics, University of Toronto, 60 St. George Street, Toronto, ON M5S 1A7, Canada

\section{INTRODUCTION}

Much of the bolometric luminosity produced by entire galaxies is emitted as thermal radiation from dust grains in the interstellar medium (ISM) at temperatures of several tens of Kelvin. The heat source is predominantly optical and ultraviolet (UV) light from stars, as well as matter accretion onto an active galactic nucleus (AGN) in some objects. In either case, the spectrum of this light tends to peak in the range $\sim 60-200 \mu \mathrm{m}$. Since the launch of the Infrared Astronomical Satellite (IRAS) in 1983, observations at these wavelengths have been essential in a number of fields, ranging from the studies of the earliest stages of star formation in dusty molecular clouds in our own Galaxy, to the detection of distant star-forming galaxies that emit most of their light in this previously unexploited band. Furthermore, excluding light from the cosmic microwave background (CMB), it is known that approximately half of all of the light in the universe is emitted at these wavelengths, the cosmic Infrared background (CIB; Puget et al. 1996; Fixsen et al. 1998, see also Devlin et al. 2009).
Observations from the ground are impossible at wavelengths in the range $\sim 60-200 \mu \mathrm{m}$, the far infrared (FIR) band, due primarily to the absorption and emission from atmospheric water vapor. Therefore, most of the progress at these wavelengths since IRAS has also been undertaken by satellites: the Infrared Space Observatory (ISO, the Spitzer Space Telescope, and the Akari Space Telescope). However, the lack of data at longer wavelengths can bias the interpretation of these results toward the assumption of warmer dust temperatures, resulting in underestimates of luminosity and mass. At slightly longer wavelengths, 350-1000 $\mu \mathrm{m}$, the submillimeter band, several spectral windows are available from dry, high-altitude sites such as Mauna Kea or the Atacama Desert. In particular, studies at $850 \mu \mathrm{m}$ with the Submillimeter Common-User Bolometer Array (SCUBA; e.g., Smail et al. 1997; Hughes et al. 1998; Holland et al. 1999; Eales et al. 2000; Dunne \& Eales 2001; Scott et al. 2002; Mortier et al. 2005; Stevens et al. 2005; Nutter \& Ward-Thompson 2007) have been used to detect very cool dust, both in our Galaxy, and in more distant galaxies. Typically, in these surveys a dust temperature must be assumed in order to estimate redshifts and luminosities, because data are available 
only at a single wavelength. Therefore, the combination of ground-based submillimeter observations with space-based FIR measurements has been required to assess the bolometric luminosities and apparent dust temperatures of everything from individual pre-stellar objects, up to entire galaxies at redshifts $z>1$. However, the general lack of high signal-to-noise data in the crucial wavelength range $\sim 200-500 \mu \mathrm{m}$ results in large uncertainties in these quantities.

In this paper, we present results from a study at 250, 350, and $500 \mu \mathrm{m}$ of several nearby galaxies using the $2 \mathrm{~m}$ Balloon-borne Large Aperture Submillimeter Telescope (BLAST). BLAST was built to fill the wavelength gap between ground-based submillimeter and space-based FIR instrumentation, using a prototype of the SPIRE camera for Herschel (Griffin et al. 2007).

In 2005 June, BLAST was launched from Esrange, Sweden, for its first scientific flight, hereafter BLAST05. It acquired data for $100 \mathrm{hr}$ before landing on Victoria Island, Canada. During this flight, BLAST observed the nearby galaxy NGC 4565. In 2006 December, BLAST conducted its second science flight, BLAST06, carrying out $250 \mathrm{hr}$ of observations above Antarctica. Six nearby galaxies were observed during BLAST06: NGC 1097, NGC 1291, NGC 1365, NGC 1512, NGC 1566, and NGC 1808.

All these galaxies are resolved by BLAST. The full widths at half-maximum (FWHM) of the BLAST06 beams are 36, 42, and $60^{\prime \prime}$ at 250, 350, and $500 \mu \mathrm{m}$ respectively. The BLAST05 flight suffered from degradation of the warm optics (Truch et al. 2008). The single galaxy in our sample from BLAST05 is nevertheless resolved, despite the large, non-Gaussian pointspread functions due to the degraded optics during this flight. We are therefore able to map the temperature and luminosity distributions, and hence dust column density, with greater signal-to-noise ratio $(\mathrm{S} / \mathrm{N})$ than any existing work. Our study can be considered a precursor to The Herschel Reference Survey (Boselli et al. 2008) and the James Clerk Maxwell Telescope (JCMT) Nearby Galaxies Legacy Survey (Wilson et al. 2009) that will be undertaken by Herschel and SCUBA-2, respectively. A complete description of the BLAST instrument is given in Pascale et al. (2008).

The known correlation between spectral energy distribution (SED) shape and luminosity in IRAS galaxies (e.g., Soifer $\&$ Neugebauer 1991) means good measurements of these quantities for rest-frame galaxies are required to put proper constraints on high-redshift FIR source count models (e.g., E. L. Chapin et al. 2010, in preparation). BLAST samples the Rayleigh-Jeans side of thermal emission from local galaxies; when combined with FIR measurements from other instruments, the dust thermal emission peak is bracketed, allowing robust determination of these quantities.

Resolved studies of galaxies are required to better model galactic structure and dynamics. The unprecedented ability of BLAST to make high-resolution maps on large angular scales in the submillimeter band provides the best opportunity to-date to study the structure of diffuse dust in nearby galaxies. To properly estimate star formation rates from submillimeter fluxes of unresolved, high redshift galaxies, an understanding of the fraction of submillimeter flux originating from AGN-driven dust heating is required. Although BLAST is unable to distinguish AGN-driven dust heating from nuclear starburst-driven heating, and this study involves nearby spirals, rather than high-redshift starbursts, we are still able to put limits on this AGN fraction by comparing flux measured from the core of these resolved galaxies with their total integrated flux. Furthermore, because
Table 1

Physical Parameters of BLAST Nearby Galaxies

\begin{tabular}{cccccc}
\hline \hline Galaxy & $\begin{array}{c}\text { R.A. } \\
(\mathrm{J} 2000)\end{array}$ & $\begin{array}{c}\text { Decl. } \\
(\mathrm{J} 2000)\end{array}$ & $\begin{array}{c}\text { Dist. } \\
(\mathrm{Mpc})\end{array}$ & Type $^{\mathrm{a}}$ & Nucleus $^{\circ}$ \\
\hline NGC 1097 & $2^{\mathrm{h}} 46^{\mathrm{m}} 19^{\mathrm{s}} 0$ & $-30^{\circ} 16^{\prime} 30^{\prime \prime}$ & $16.9^{\mathrm{b}}$ & SB(s)b & Sy1 \\
NGC 1291 & $3^{\mathrm{h}} 17^{\mathrm{m}} 18^{\mathrm{s}} 6$ & $-41^{\circ} 06^{\prime} 29^{\prime \prime}$ & $9.7^{\mathrm{b}}$ & $(\mathrm{R}) \mathrm{SB}(\mathrm{s}) 0 / \mathrm{a}$ & $\cdots$ \\
NGC 1365 & $3^{\mathrm{h}} 33^{\mathrm{m}} 36^{\mathrm{s}} 4$ & $-36^{\circ} 08^{\prime} 25^{\prime \prime}$ & $18.4^{\mathrm{c}}$ & SB(s)b & Sy2 \\
NGC 1512 & $4^{\mathrm{h}} 03^{\mathrm{m}} 54.3$ & $-43^{\circ} 20^{\prime} 56^{\prime \prime}$ & $10.4^{\mathrm{b}}$ & SB(r)a & $\cdots$ \\
NGC 1566 & $4^{\mathrm{h}} 20^{\mathrm{m}} 00^{\mathrm{s}} .4$ & $-54^{\circ} 56^{\prime} 16^{\prime \prime}$ & $18.0^{\mathrm{b}}$ & SAB(s)bc & Sy1 \\
NGC 1808 & $5^{\mathrm{h}} 07^{\mathrm{m}} 42^{\mathrm{s}} .3$ & $-37^{\circ} 30^{\prime} 47^{\prime \prime}$ & $10.9^{\mathrm{d}}$ & $(\mathrm{R}) \mathrm{SAB}(\mathrm{s}) \mathrm{a}$ & Sy2 \\
NGC 4565 & $12^{\mathrm{h}} 36^{\mathrm{m}} 20.8$ & $+25^{\circ} 59^{\prime} 16^{\prime \prime}$ & $12.6^{\mathrm{e}}$ & SA(s)b & Sy2 \\
\hline
\end{tabular}

Notes.

a RC3 morphological type from de Vaucouleurs et al. (1991).

b Kennicutt et al. (2003).

c Komossa \& Schulz (1998).

d Jiménez-Bailón et al. (2005).

e Lawrence et al. (1999).

BLAST can make high-fidelity large-area maps, it is able to measure the complete emission from these spatially extended galaxies.

\section{DATA REDUCTION}

The common data reduction pipeline for BLAST has been presented in Truch et al. (2008) and Patanchon et al. (2008). Maps used in this analysis for NGC 4565 were made using the maximum-likelihood map maker SANEPIC (Patanchon et al. 2008), which was written explicitly for BLAST analysis.

Maps of BLAST observations of the remaining galaxies were produced using ALMAGEST (Wiebe 2008), an iterative, maximum-likelihood map maker also written for BLAST analysis, and algorithmically similar to other iterative map makers used in CMB analysis (e.g., Prunet et al. 2000). Because most of these galaxies were observed only at a single scan direction, and hence lack cross-linking, the maps produced are artificially constrained to zero flux far from the observed galaxy to improve convergence properties of the iterative algorithm (see Section 3.8 of Patanchon et al. 2008). In relatively high $\mathrm{S} / \mathrm{N}$ cases, such as these galaxy maps, ALMAGEST output is consistent with maps made with SANEPIC (Wiebe 2008), but may be produced faster, and allow arbitrary constraints to be applied simply.

\section{OBSERVATIONS}

\subsection{BLAST Observations}

The seven nearby galaxies observed by BLAST were selected based on a number of competing criteria. First, the visibility requirements were restrictive, as they took into account BLAST's limited elevation range $\left(25^{\circ}-60^{\circ}\right)$, Sun and Moon avoidance criteria, and still had to make allowance for the changing latitude and longitude of the telescope arising from BLAST's uncertain ground path while aloft. Second, the galaxies had to be sufficiently large to be resolved by BLAST's optics (e.g., BLAST's $36^{\prime \prime}$ beam size at $250 \mu \mathrm{m}$ corresponds to $\sim 2.5 \mathrm{kpc}$ at the average distance of $14 \mathrm{Mpc}$ for this galaxy sample). Third, they had to be sufficiently luminous for BLAST to be able to map them with a high $\mathrm{S} / \mathrm{N}$ in the relatively small fraction of the flights scheduled for these observations. Finally, the sample needed to have measurements from the IRAS, the Spitzer Space Telescope, SCUBA, or other experiments complementary to BLAST. 
Table 2

Observed Flux Densities and Derived Quantities for BLAST Nearby Galaxies

\begin{tabular}{|c|c|c|c|c|c|}
\hline Galaxy & $\begin{array}{c}250 \mu \mathrm{m} \\
(\mathrm{Jy})\end{array}$ & $\begin{array}{c}350 \mu \mathrm{m} \\
(\mathrm{Jy})\end{array}$ & $\begin{array}{c}500 \mu \mathrm{m} \\
(\mathrm{Jy})\end{array}$ & $\begin{array}{l}T_{\mathrm{d}} \\
(\mathrm{K}) \\
\end{array}$ & $\log \left(\frac{M_{\mathrm{d}}}{M_{\odot}}\right)$ \\
\hline NGC 1097 & $89.4 \pm 0.2 \pm 7.7$ & $40.5 \pm 0.1 \pm 3.0$ & $16.2 \pm 0.1 \pm 1.4$ & $21.3 \pm 0.3$ & $\ldots{ }^{a}$ \\
\hline NGC 1291 & $26.1 \pm 0.2 \pm 2.6$ & $12.7 \pm 0.1 \pm 1.0$ & $6.4 \pm 0.1 \pm 0.5$ & $16.6 \pm 0.4$ & $\ldots$ a \\
\hline NGC 1365 & $145.8 \pm 0.3 \pm 12.9$ & $62.3 \pm 0.2 \pm 4.6$ & $24.7 \pm 0.1 \pm 2.1$ & $19.9 \pm 0.4$ & $8.72_{-0.10}^{+0.08}$ \\
\hline NGC 1512 & $14.7 \pm 0.1 \pm 1.3$ & $6.9 \pm 0.1 \pm 0.5$ & $3.4 \pm 0.1 \pm 0.3$ & $20.3 \pm 0.3$ & \\
\hline NGC 1566 & $51.5 \pm 0.2 \pm 4.6$ & $22.2 \pm 0.1 \pm 1.7$ & $9.1 \pm 0.1 \pm 0.8$ & $20.1 \pm 0.4$ & $\ldots{ }^{a}$ \\
\hline NGC 1808 & $84.0 \pm 0.1 \pm 7.1$ & $33.1 \pm 0.1 \pm 2.5$ & $11.8 \pm 0.1 \pm 1.0$ & $22.8 \pm 0.5$ & $7.85_{-0.11}^{+0.09}$ \\
\hline NGC 4565 & $37.2 \pm 0.5 \pm 4.5$ & $21.0 \pm 0.3 \pm 2.1$ & $9.8 \pm 0.3 \pm 0.8$ & $16.1_{-0.5}^{+0.4}$ & $8.21_{-0.11}^{+0.09}$ \\
\hline
\end{tabular}

Notes. Flux uncertainties reported are first the measurement error $\left(\sigma_{\mathrm{d}}\right)$ and then the calibration error $\left(\sigma_{\mathrm{c}}\right)$. Temperatures and dust masses are extracted from the modified blackbody fits presented in Figure 8.

a This modified blackbody fit is used to calculate $\kappa$ from the DL07 model fit, so no independent mass is determined.

Table 1 summarizes relevant physical parameters of the BLAST nearby galaxy sample. ${ }^{15}$ The measured total flux density in each BLAST band is tabulated in Table 2.

\subsection{Other Multiwavelength Data}

As part of the Spitzer Infrared Nearby Galaxies Survey (SINGS; Kennicutt et al. 2003), four galaxies in our sample (NGC 1097, NGC 1291, NGC 1512, and NGC 1566, hereafter BLAST/SINGS galaxies) have also been observed by the Multiband Imaging Photometer for Spitzer (MIPS) at $160 \mu \mathrm{m}$, $70 \mu \mathrm{m}$, and $24 \mu \mathrm{m}$ (Rieke et al. 2004) and the Infrared Array Camera (IRAC) at $8 \mu \mathrm{m}, 6 \mu \mathrm{m}, 4.5 \mu \mathrm{m}$, and $3.6 \mu \mathrm{m}$ (Fazio et al. 2004), both on the Spitzer Space Telescope. The remaining galaxies in our sample were not observed by Spitzer. Spatially integrated Spitzer fluxes are taken from Dale et al. (2007), and MIPS maps used in this analysis come from the fifth SINGS public data release. ${ }^{16}$

IRAS data for all of our sample except NGC 1291 have been extracted from SCANPI. ${ }^{17} I R A S$ data for the fainter and more extended NGC 1291 are measured from High Resolution Echelle Spectrometer generated maps (Aumann et al. 1990). These IRAS data are used, in addition to Spitzer, to constrain the spatially integrated SEDs of these galaxies. A $20 \%$ uncorrelated uncertainty is applied to all IRAS data. IRAS $25 \mu \mathrm{m}$ data are not used in this analysis when equivalent MIPS $24 \mu \mathrm{m}$ data are available.

Two of our sample have been observed by SCUBA: NGC 1097 at $850 \mu \mathrm{m}$ (Dale et al. 2005), and NGC 1808 at both 450 and $850 \mu \mathrm{m}$ (Stevens et al. 2005). These measurements do not cover the entire region of emission detected by BLAST and we omit these measurements from our fits for this reason.

For NGC 1808, we calculate aperture corrections for the Stevens et al. (2005) reported flux densities. We apply the Stevens et al. apertures to the BLAST observations and compare the $500 \mu \mathrm{m}$ flux inside these apertures to the total $500 \mu \mathrm{m}$ flux. From this, we calculate flux densities for NGC 1808 of $s_{450}=13.12$ Jy and $s_{850}=1.76 \mathrm{Jy}$ by applying the aperture corrections to the SCUBA measurements. These values are consistent with our $\beta=2$ modified blackbody model fit to BLAST plus $100 \mu \mathrm{m}$ IRAS (Section 4.3).

\footnotetext{
15 Instructions for obtaining BLAST maps used in this analysis may be found on http://blastexperiment.info/.

16 Obtained from http://data.spitzer.caltech.edu/popular/sings/.

17 Available at http://scanpi.ipac.caltech.edu:9000/applications/Scanpi/ index.html.
}

We also exclude data from the ISO for NGC 1365 and NGC 4565, again because of aperture effects.

\subsection{Individual Galaxies}

A brief description of each observed galaxy is given as follows.

\subsubsection{NGC 1097}

Features of NGC 1097, presented in Figure 1, include a bar $20 \mathrm{kpc}$ in length that tapers off into two spiral arms (Beck et al. 2005), as well as several fringe structures called jets or "rays" (Wolstencroft \& Zealey 1975; Lorre 1978). NGC 1097 has an active Seyfert 1/LINER nucleus, which is considered to have a low luminosity (Mason et al. 2007), and is surrounded by a $1.5 \mathrm{kpc}$ diameter nuclear ring, containing $1.1 \times 10^{9} M_{\odot}$ of molecular gas (Hsieh et al. 2008; Gerin et al. 1988). Thermal dust heating in the nuclear ring is dominated by a starburst (Mason et al. 2007) with a stellar production rate of $5 M_{\odot} \mathrm{yr}^{-1}$ (Hummel et al. 1987). BLAST made two observations of a $\sim 0.25 \mathrm{deg}^{2}$ area centered on NGC 1097, with a total integration time of 52 minutes. BLAST clearly resolves the bar and spiral arms of the galaxy, but has insufficient resolution to resolve the $1.5 \mathrm{kpc}$ star-forming ring.

\subsubsection{NGC 1291}

NGC 1291, presented in Figure 2, is the brightest Sa galaxy in the Shapley-Ames Catalogue (Bregman et al. 1995). NGC 1291 has an orientation that is almost "face on" and has a primary bar and a secondary bar that have a $30^{\circ}$ misalignment with each other (Pérez \& Freeman 2006). The total $\mathrm{H}$ I mass is $2.4 \times 10^{9} M_{\odot}$, and there is little evidence for star formation in the central bulge (Hogg et al. 2001). BLAST made four observations of a $\sim 0.4 \mathrm{deg}^{2}$ area centered on NGC 1291, with a total integration time of 92 minutes. Although diffuse, BLAST detects both the central core of the galaxy, as well as the ring-like spiral structure $4^{\prime}$ from the core.

\subsubsection{NGC 1365}

NGC 1365, presented in Figure 3, is usually classified as a Seyfert 1.5, but some authors list it as Seyfert 1 or Seyfert 2 (e.g., Galliano et al. 2005; Véron et al. 1980; Alloin et al. 1981). The AGN has low luminosity, but there is evidence from optical studies to suggest that there is star formation surrounding the nucleus (Komossa \& Schulz 1998). BLAST made three observations of a $\sim 0.25 \mathrm{deg}^{2}$ area centered on NGC 1365, with a total integration time of 30 minutes. The brightest of our 


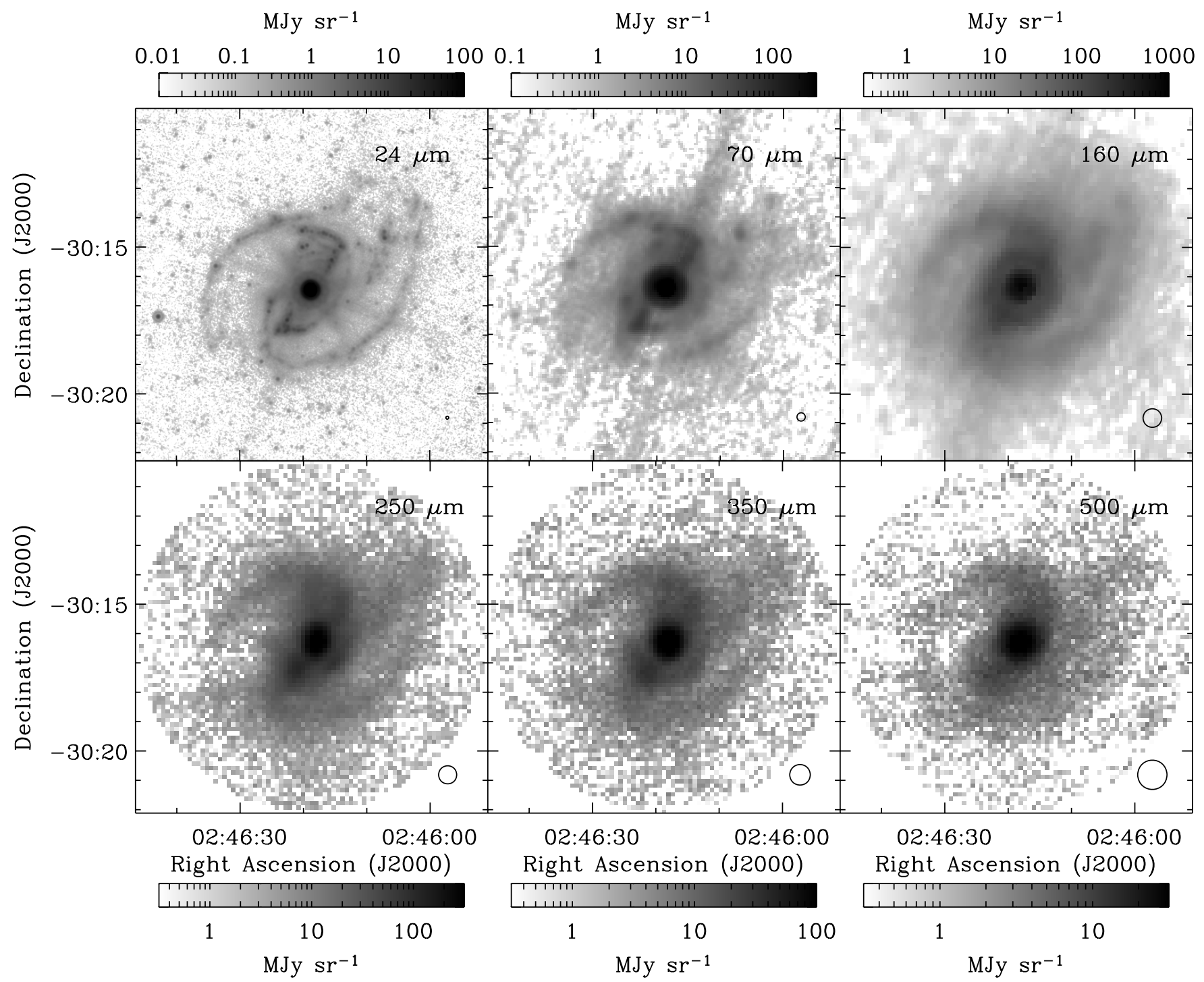

Figure 1. BLAST (bottom row) and MIPS (top row) observations of NGC 1097. The radius of the clipped BLAST map is $6{ }^{\prime}$. The intensity scale is logarithmic. The FWHM size of the BLAST and MIPS beams are plotted for reference in the lower right corner. BLAST resolves the spiral arms and central bar of the galaxy. The $24 \mu \mathrm{m}$ image is shown for comparison only, and not used in this analysis.

sample, BLAST's observations prominently show both the bar and spiral arm structure of the galaxy.

\subsubsection{NGC 1512}

NGC 1512, presented in Figure 4, has a nucleus surrounded by a circumnuclear ring (Maoz et al. 2000). Beyond the ring, its spiral arms are complicated by its satellite galaxy, NGC 1510 (Kinman 1978). BLAST made three observations of a $\sim 0.45 \mathrm{deg}^{2}$ area centered on NGC 1512, with a total integration time of 28 minutes. BLAST detects the core and tightly wound central spiral of NGC 1512. BLAST also detects the companion galaxy, NGC 1510.

\subsubsection{NGC 1566}

NGC 1566, presented in Figure 5, is the second brightest known Seyfert galaxy (de Vaucouleurs 1973). Extending from the nucleus are two prominent spiral arms that continue outward to form the galaxy's outer ring that is at an inclination of about $30^{\circ}$ (de Vaucouleurs 1975; Bottema 1992). The arms contain a significant amount of star formation (Bottema 1992).
BLAST made two observations of a $\sim 0.7 \mathrm{deg}^{2}$ area centered on NGC 1566, with a total integration time of 50 minutes. BLAST resolves the spiral structure of the galaxy, and the observations are dominated by submillimeter flux originating in the core of the galaxy.

\subsection{6. $N G C 1808$}

NGC 1808, presented in Figure 6, is a Seyfert 2 galaxy (Jiménez-Bailón et al. 2005). Subsequent measurement seems to indicate that a large part of the radiation emanating from the central $1 \mathrm{kpc}$ of the galaxy is from an AGN as well as a high rate of star formation (Förster Schreiber et al. 2003; Maiolino et al. 2003). Using ROSAT, Junkes et al. (1995) estimated a star formation rate of between 5 and $13 M_{\odot} \mathrm{yr}^{-1}$, and a supernova event rate of $0.09 \mathrm{yr}^{-1}$. A companion galaxy, not observed by BLAST, NGC 1792 is located 40' from NGC 1808 (130 kpc at a distance of $10.9 \mathrm{Mpc}$ ) and may be responsible for an accelerated star formation rate in NGC 1808 (Jiménez-Bailón et al. 2005). BLAST made four observations of a $\sim 1 \mathrm{deg}^{2}$ area centered on NGC 1808, with a total integration time of 179 minutes. BLAST 


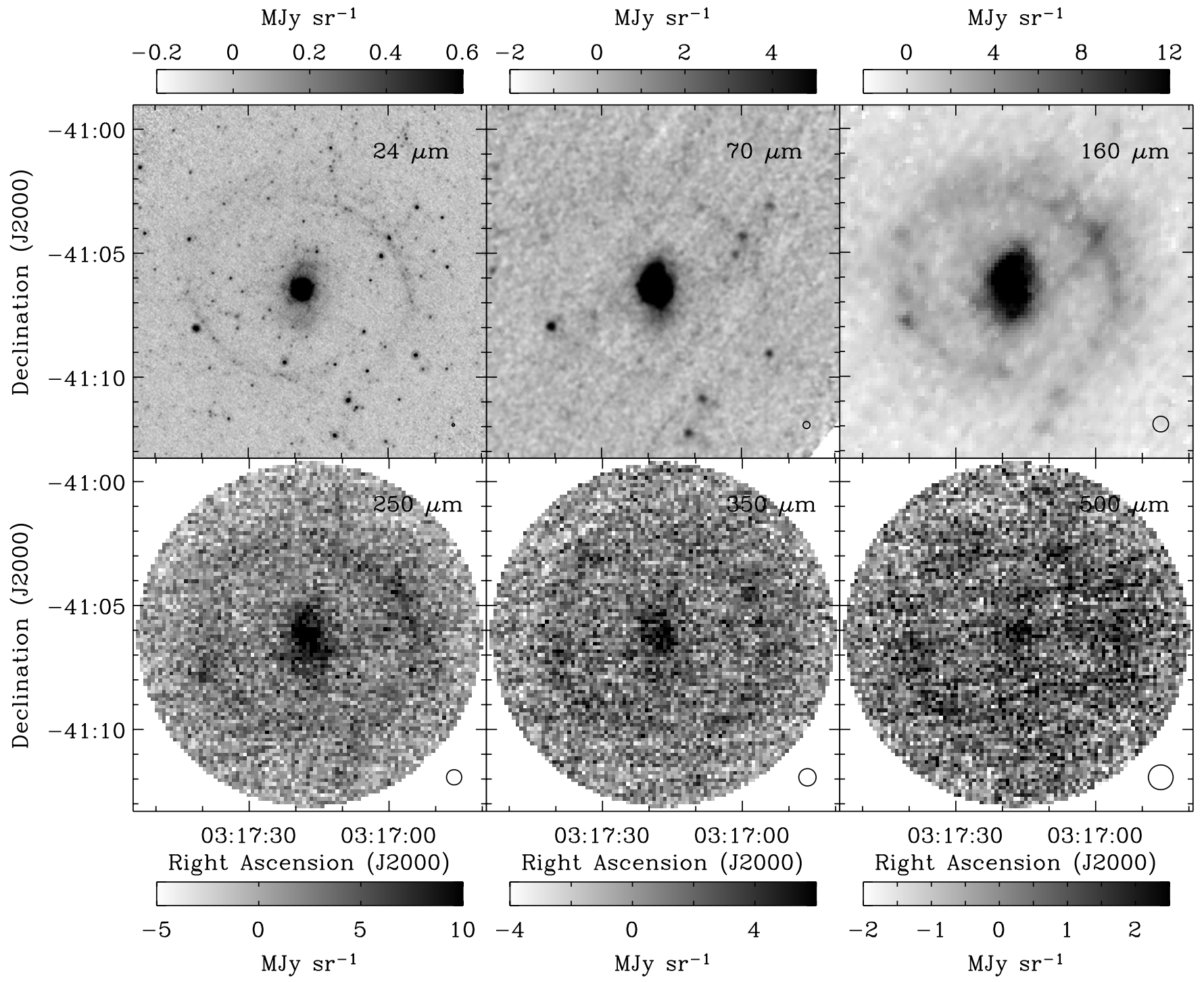

Figure 2. BLAST (bottom row) and MIPS (top row) observations of NGC 1291. The radius of the clipped map BLAST map is $7^{\prime}$. The intensity scale is linear. The size of the BLAST and MIPS beams are plotted for reference in the lower right corner. The outer $4^{\prime}$ ring of spiral arm structure is detected in all three BLAST bands, as is the central core.

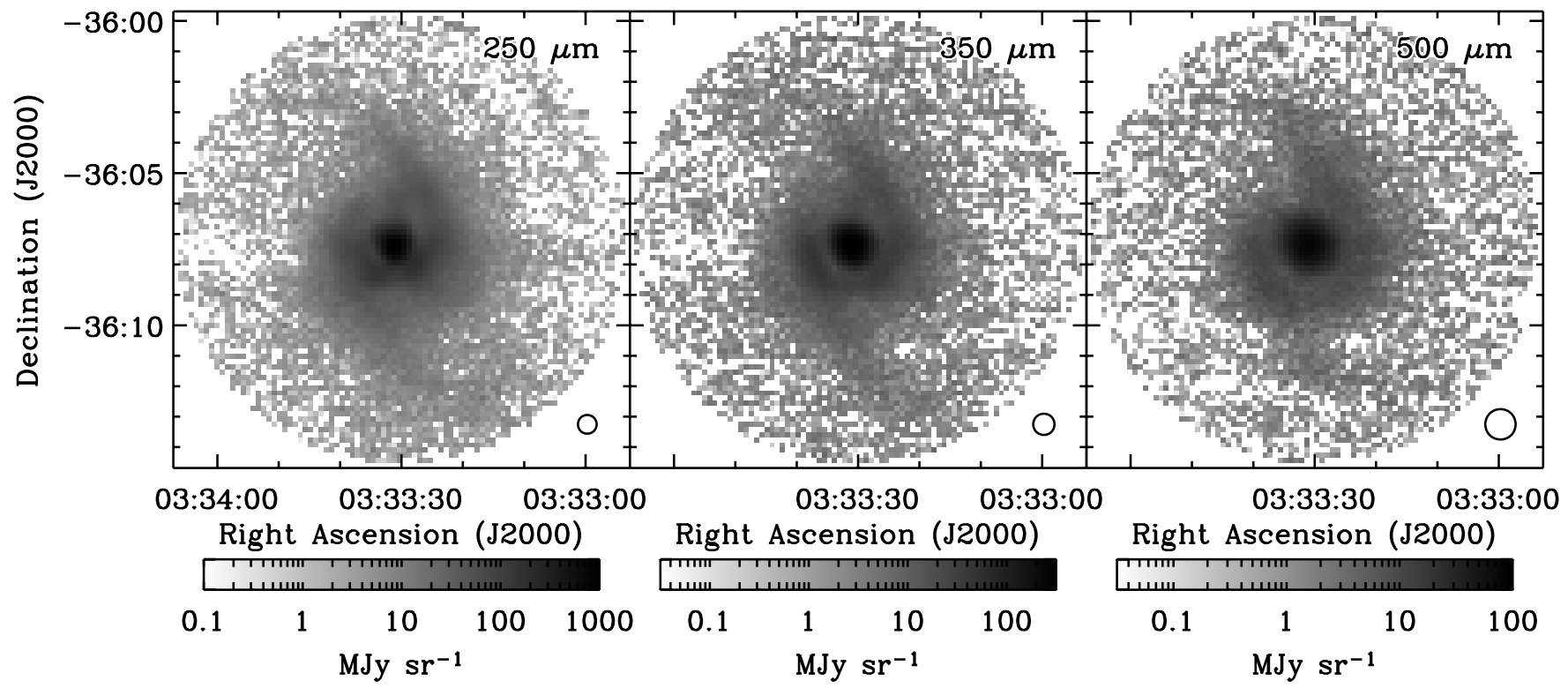

Figure 3. BLAST observations of NGC 1365. Pixels are 9" on a side. The radius of the clipped map is 6.5. The intensity scale is logarithmic. 


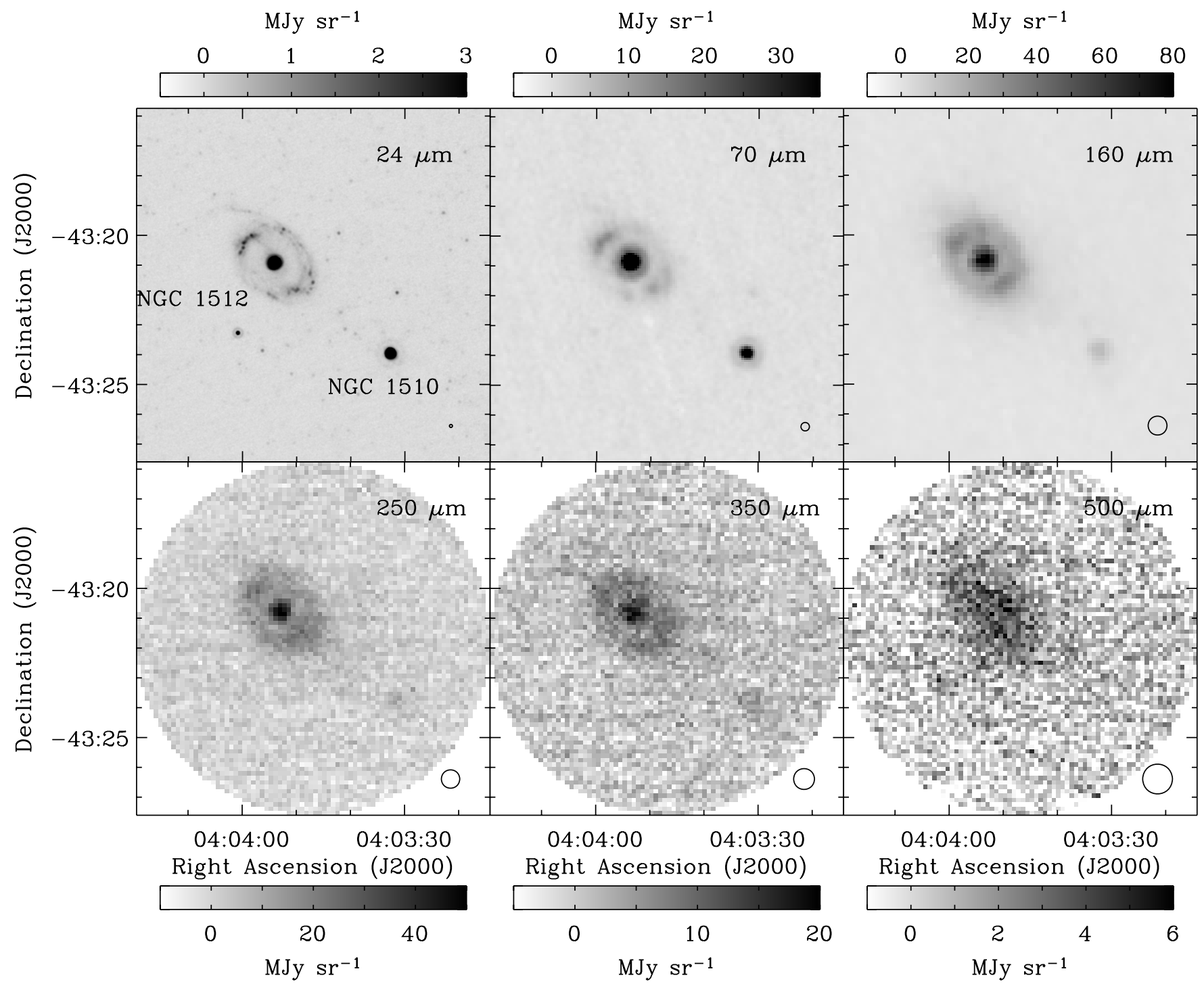

Figure 4. BLAST (bottom row) and MIPS (top row) observations of NGC 1512. The radius of the clipped BLAST maps is $6^{\prime}$. The intensity scale is linear. The size of the BLAST and MIPS beams are plotted for reference in the lower right corner. BLAST detects the companion galaxy, NGC $1510\left(\alpha=4^{\mathrm{h}} 03^{\mathrm{m}} 33^{\mathrm{s}}, \delta=-43^{\circ} 24^{\prime}\right.$; lower right) at $250 \mu \mathrm{m}$ and $350 \mu \mathrm{m}$. In these two bands, BLAST can also distinguish between the core and disk of NGC 1512.

detects both the core and the disk of the galaxy. BLAST also detects excess flux in this map near the location of the interacting galaxies ESO 305-IG $010\left(\alpha=5^{\mathrm{h}} 08^{\mathrm{m}} 27^{\mathrm{s}}, \delta=-37^{\circ} 39^{\prime} \cdot 5\right.$, $z=0.0524)$

\subsection{7. $N G C 4565$}

NGC 4565, presented in Figure 7, is an edge-on barredspiral galaxy in the constellation Coma. It has an apparent size of about $12^{\prime} \times 2.5$. Although degraded by the large pointspread functions of BLAST05 (186-189", Truch et al. 2008), NGC 4565 is still resolved by BLAST. BLAST made one observation of a $\sim 0.4 \mathrm{deg}^{2}$ area centered on NGC 4565 , with a total integration time of 49 minutes. A beam-deconvolved image, using the method described in Section 2.4 of Chapin et al. (2008), is shown in the bottom row of Figure 7.

We fit an exponential profile to the beam-deconvolved maps of the form $\exp (|x| / s)$, where $x$ is a spatial variable aligned along the major axis of the galactic disk and $s$ is the scale length, and find that $s=118^{\prime \prime}, 156^{\prime \prime}$, and $142^{\prime \prime}$ at $250 \mu \mathrm{m}, 350 \mu \mathrm{m}$, and $500 \mu \mathrm{m}$, respectively. The $250 \mu \mathrm{m}$ fit, which has the highest $\mathrm{S} / \mathrm{N}$, agrees well with scale lengths measured at other wave- lengths (e.g., van der Kruit \& Searle 1981; Engargiola \& Harper 1992; Rice et al. 1996). In this fit, we have ignored the central $3^{\prime}$ region of the galaxy. This region corresponds to an area of reduced $250 \mu \mathrm{m}$ flux density, possibly corresponding to the molecular ring seen in ${ }^{12} \mathrm{CO}$ (Neininger et al. 1996).

\section{SED FITTING}

\subsection{Physical Dust Models}

For the four BLAST/SINGS galaxies, we fit the silicategraphite-polycyclic aromatic hydrocarbon (PAH) dust models of Draine \& Li (2007), hereafter DL07, to BLAST, MIPS, IRAS, and IRAC data. These models have already been applied, without the benefit of BLAST observations, to the same galaxies in Draine et al. (2007), hereafter Dr07. We independently recreate this analysis with the inclusion of the BLAST data. These models provide the dust emissivity per hydrogen atom, $j_{v}\left(q_{\mathrm{PAH}}, U_{\min }, U_{\mathrm{max}}\right)$, dependent on three model parameters:

1. $q_{\mathrm{PAH}}$ : the fraction of total dust mass in PAHs containing less than $10^{3}$ carbon atoms, 


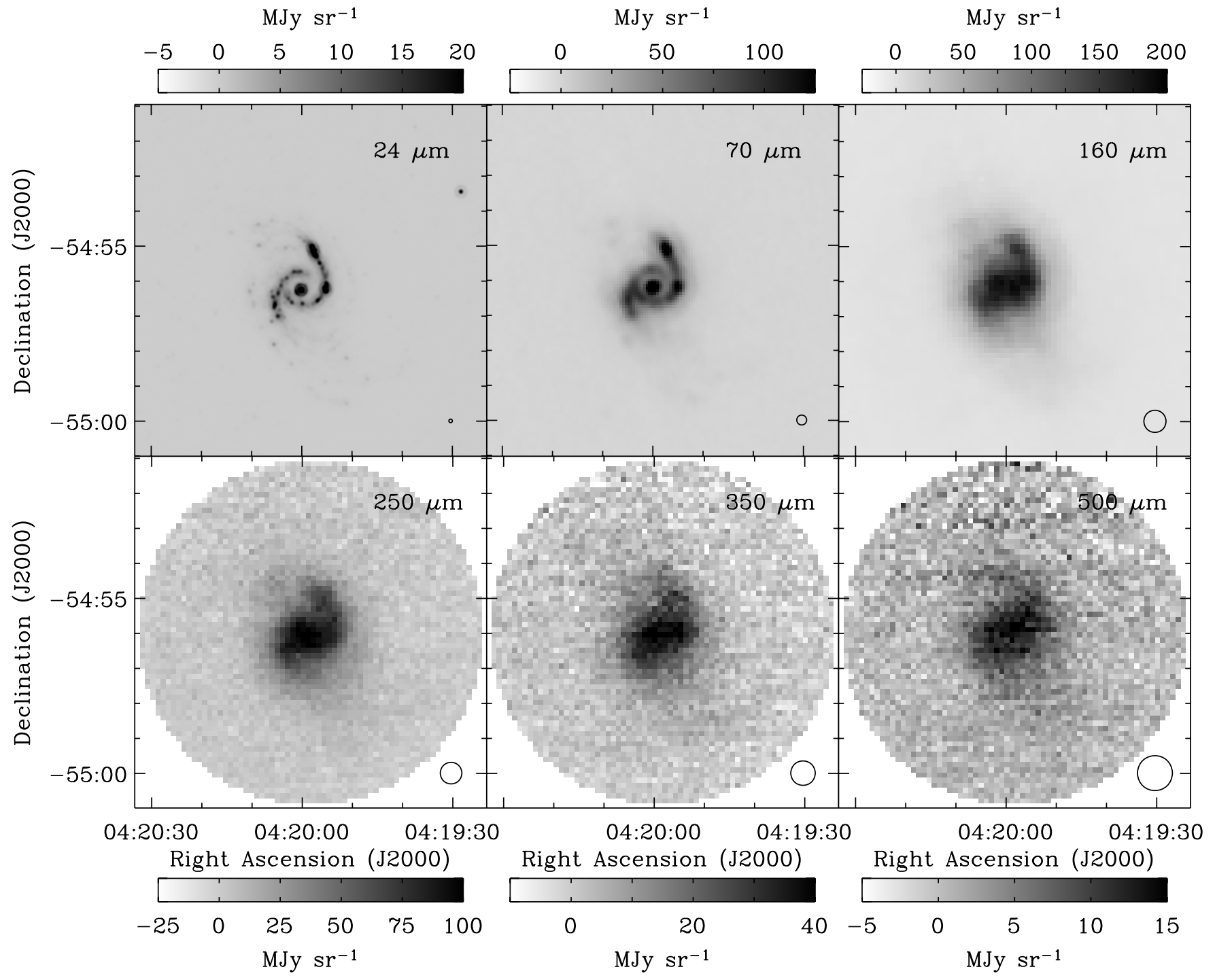

Figure 5. BLAST (bottom row) and MIPS (top row) observations of NGC 1566 . The radius of the clipped BLAST maps is $5^{\prime}$. The intensity scale is linear. The size of the BLAST and MIPS beams are plotted for reference in the lower right corner. BLAST detects the spiral arms, although the map is dominated by flux from the core.

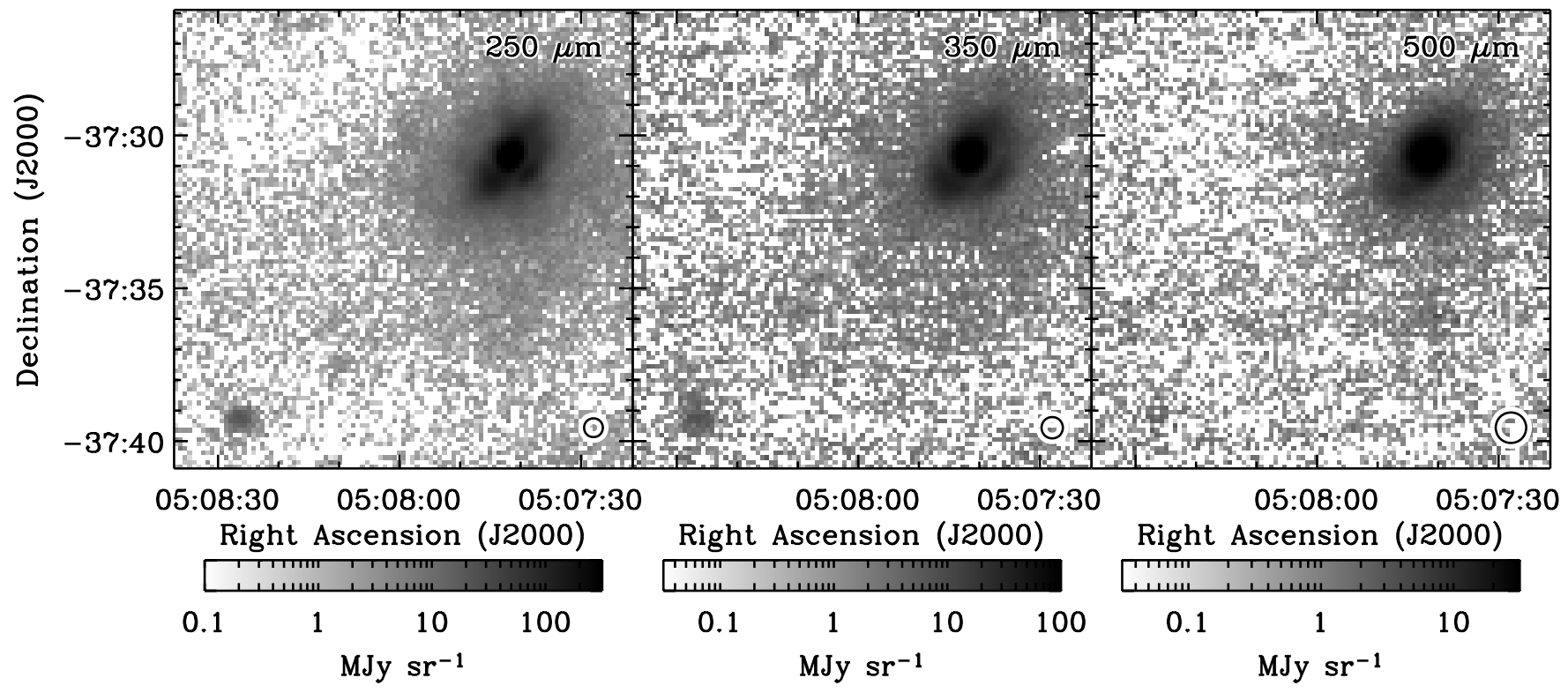

Figure 6. BLAST observations of NGC 1808 (upper right). Pixels are $9^{\prime \prime}$ on a side. BLAST also detects excess flux density near the position of the interacting galaxies ESO 305-IG $010\left(\alpha=5^{\mathrm{h}} 08^{\mathrm{m}} 27^{\mathrm{s}}, \delta=-37^{\circ} 39^{\prime} \cdot 5, z=0.0524\right.$; lower left $)$. The intensity scale is logarithmic. 


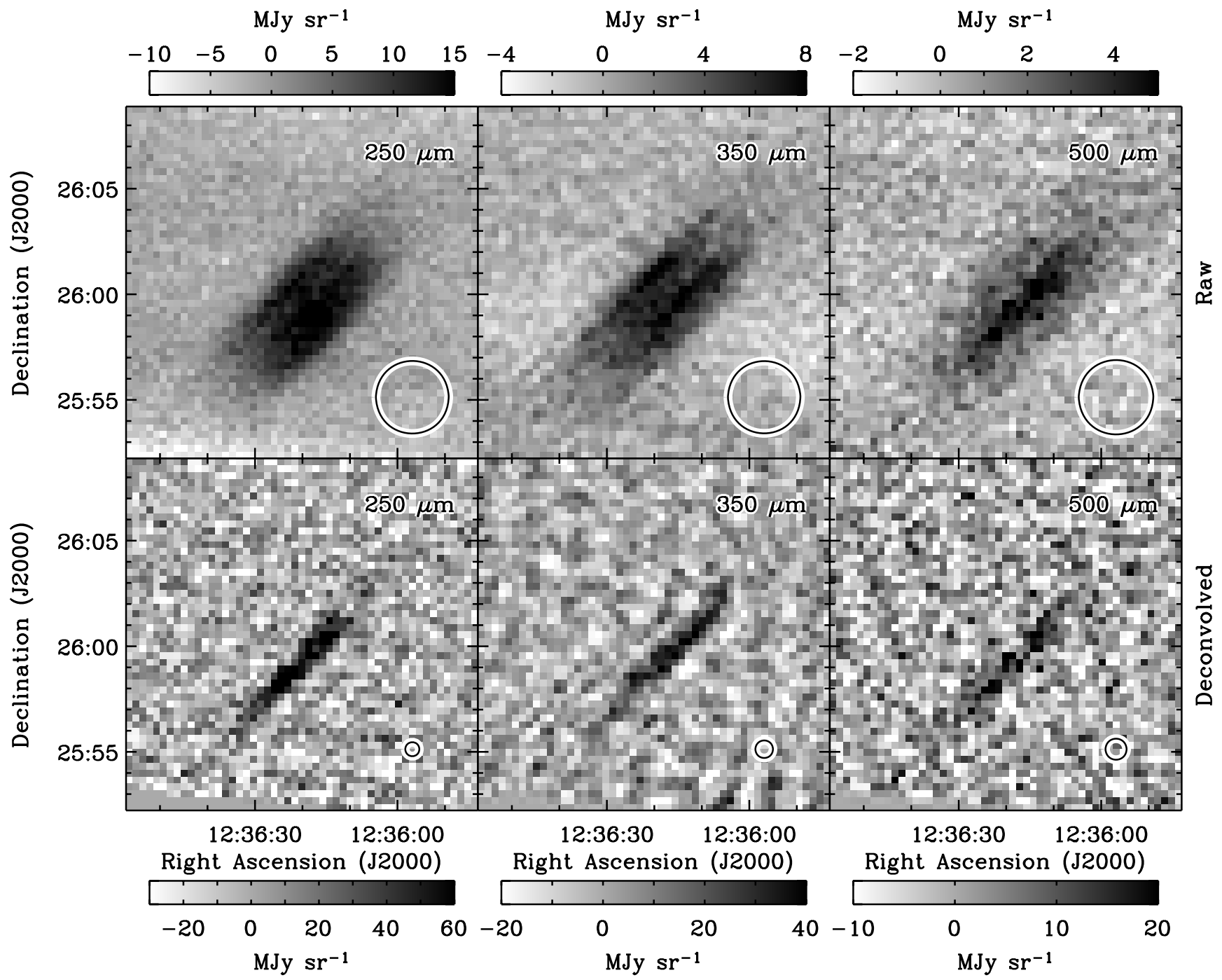

Figure 7. Top row: BLAST observations of NGC 4565. Bottom row: results of deconvolving the raw maps following the procedure outlined in Section 2.4 of Chapin et al. (2008). This deconvolution process results in 3-5 times better resolution, at a cost of reduction in signal to noise of a factor of $\sim 2$. Pixels are 20" on a side. Note the linear intensity scale. Effective beam sizes, based on the full widths at half-power (FWHP) found in Table 1 of Chapin et al. (2008), are plotted in the lower right corners for both the raw and deconvolved maps. The full BLAST05 point-spread functions are shown in Figure 1 of Truch et al. (2008).

2. $U_{\min }$ : a starlight intensity factor characterizing the radiation field heating the diffuse ISM,

3. $U_{\max }$ : a starlight intensity factor characterizing the radiation field in more intense star-forming regions, including photodissociative regions (PDRs).

Both $U_{\min }$ and $U_{\max }$ are relative to the specific energy density of starlight measured by Mathis et al. (1983). Dr07 consider a fourth model parameter, $\alpha$, which is the exponent characterizing the power-law decrease in starlight intensity: $d M_{\mathrm{d}} / d U \propto U^{-\alpha}$. They found the model fits to be insensitive to its value and the models provided have $\alpha=2$.

Our fitting procedure follows that of Dr07. We restrict ourselves to the seven Milky Way dust model sets ${ }^{18}$ (Weingartner \& Draine 2001; updated by DL07). These seven model sets have values of $q_{\mathrm{PAH}}$ between $0.47 \%$ and $4.6 \%$. We take linear combinations of these to produce 43 different model sets for a finer-grained sampling of $q_{\mathrm{PAH}}$. Each set contains values of $j_{v}$ for 22 values of $U_{\min }$ between 0.1 and 25 , and for 5 "PDR models" with values of $U_{\max }\left(10^{2}, 10^{3}, 10^{4}, 10^{5}, 10^{6}\right)$, plus one "diffuse ISM model" with $U_{\max }=U_{\min }$.

\footnotetext{
18 Obtained from http://www.astro.princeton.edu/ draine/dust/dust.html.
}

These dust models are combined with a stellar blackbody to produce a galactic model:

$$
\begin{aligned}
F_{\nu, \text { model }}= & \Omega_{\star} B_{v}\left(T_{\star}\right)+\frac{M_{\mathrm{d}}}{m_{\mathrm{H}} D^{2}}\left(\frac{M_{\mathrm{H}}}{M_{\mathrm{d}}}\right) \\
& \times\left[(1-\gamma) j_{v}\left(q_{\mathrm{PAH}}, U_{\min }, U_{\min }\right)\right. \\
& \left.+\gamma j_{v}\left(q_{\mathrm{PAH}}, U_{\min }, U_{\max }\right)\right],
\end{aligned}
$$

where $\Omega_{\star}$ is the solid angle subtended by stars, $B_{v}(T)$ is the Planck function, the nominal stellar temperature $T_{\star}=5000 \mathrm{~K}$, $M_{\mathrm{d}}$ is the total dust mass, $m_{\mathrm{H}}$ is the mass of a hydrogen atom, $D$ is the distance to the galaxy, and $\gamma$ is a mixing fraction between the diffuse ISM $\left(U_{\max }=U_{\min }\right)$ and PDR $\left(U_{\max }>U_{\min }\right)$ dust models. The hydrogen-to-dust mass ratio, $M_{\mathrm{H}} / M_{\mathrm{d}}$, is set by the model choice, and varies from 96 to 100.

Like Dr07, we find that the best fits for the four galaxies are insensitive to the choice of $U_{\max }$, and so set $U_{\max }=10^{6}$. We obtain the best-fit values of $\Omega_{\star}, M_{\mathrm{d}}, \gamma, q_{\mathrm{PAH}}$, and $U_{\min }$ through $\chi^{2}$ minimization (see Section 4.5).

The best-fit models for the galaxies to the extended Spitzer-IRAS-BLAST data set are plotted in Figure 8. This figure also plots the spatially integrated Spitzer 


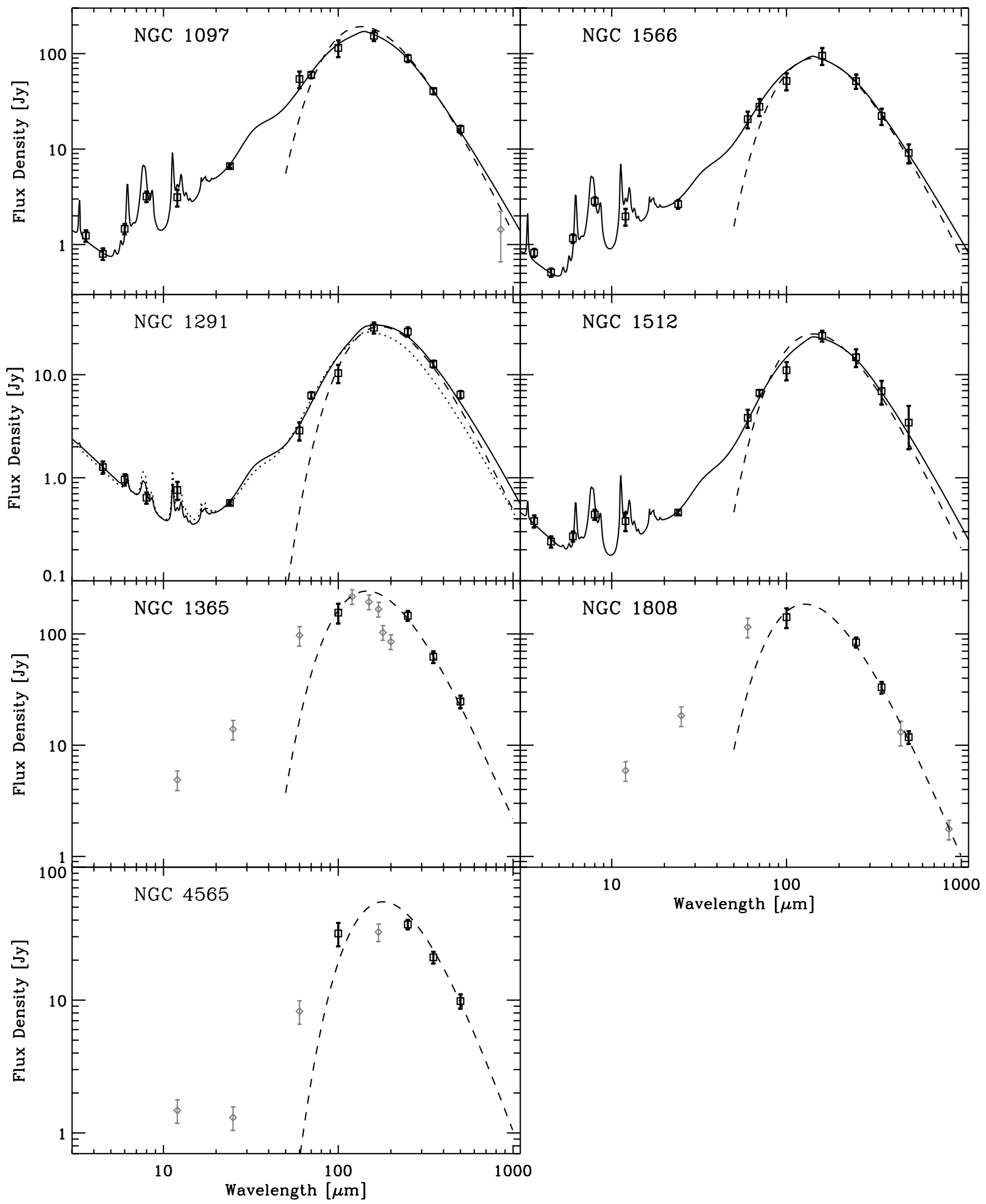

Figure 8. Spatially integrated SEDs for the seven galaxies in our sample. Data from BLAST, MIPS, IRAC, and IRAS used in this analysis are shown as black squares. Other data, which are not used in this analysis, from IRAS for NGC 1365, NGC 1808, and NGC 4565, SCUBA for NGC 1097 (Dale et al. 2005 ), and NGC 1808 (Stevens et al. 2005), and ISO for NGC 1365, and NGC 4565 (Spinoglio et al. 2002; Stickel et al. 2004) are the lighter diamonds. The aperture corrections calculated in Section 3.2 have been applied to the SCUBA measurements of NGC 1808. The solid curves are the best-fit physical dust models of Draine \& Li (2007) (DL07; see Section 4.1). The dotted curve in the panel for NGC 1291 is the best-fit DL07 model subject to the constraint $U_{\min } \geqslant 0.7$. The dashed curves are the best-fit single-component modified blackbody model (Section 4.3). Parameter values for these fits are given in Tables 2 and 3. 
Table 3

DL07 Model Parameters for BLAST Nearby Galaxies

\begin{tabular}{lcrrrrrrrc}
\hline \hline \multicolumn{1}{c}{ Galaxy } & $\log \left(\frac{M_{\mathrm{d}}}{M_{\odot}}\right)$ & $\log \left(\frac{L_{\mathrm{d}}}{L_{\odot}}\right)$ & $\begin{array}{c}q_{\mathrm{PAH}} \\
(\%)\end{array}$ & $\langle U\rangle$ & $U_{\min }$ & $\begin{array}{c}\gamma \\
(\%)\end{array}$ & $\begin{array}{c}\chi_{r}^{2} \\
\left(\mathrm{~m}^{2} \mathrm{~kg}^{-1}\right)\end{array}$ \\
\hline NGC 1097 & 8.37 & 10.80 & 3.0 & 2.00 & 1.5 & 2.80 & 0.58 & 0.299 \\
NGC 1291 & 7.62 & 9.42 & 1.6 & 0.46 & 0.4 & 1.10 & 3.47 & 0.290 \\
NGC 1291 & 7.43 & 9.45 & 3.1 & 0.77 & 0.7 & 0.70 & 5.66 & $\ldots$ \\
NGC 1512 & 7.25 & 9.42 & 3.2 & 1.10 & 1.0 & 0.80 & 0.87 & 0.247 \\
NGC 1566 & 8.21 & 10.58 & 4.6 & 1.74 & 1.5 & 1.30 & 1.13 & 0.305 \\
\hline
\end{tabular}

Notes.

a At $250 \mu \mathrm{m}$.

b With constraint $U_{\min } \geqslant 0.7$.

and IRAS, BLAST, and SCUBA measurements for these galaxies.

The model parameters are tabulated in Table 3 . We also tabulate the dust-weighted mean starlight intensity scale factor,

$$
\langle U\rangle=\left[(1-\gamma) U_{\min }+\gamma \frac{\ln \left(U_{\max } / U_{\min }\right)}{U_{\min }^{-1}-U_{\max }^{-1}}\right],
$$

and the dust luminosity calculated from the model fit.

\subsection{Modified Blackbody SEDs}

In addition to the DL07 models, we also fit BLAST plus available MIPS 70 and $160 \mu \mathrm{m}$ and IRAS $100 \mu \mathrm{m}$ data to a single-component modified blackbody SED:

$$
F_{v, \text { model }}=\frac{M_{\mathrm{d}} \kappa}{D^{2}}\left(\frac{v}{v_{0}}\right)^{\beta} B_{v}(T)
$$

where the dust emissivity index, $\beta$, is fixed to 2 , and $v_{0}$ to $1.2 \mathrm{THz}=c / 250 \mu \mathrm{m}$. An uncertainty of $\pm 0.3 \mathrm{Mpc}$ is assumed for all galaxy distances. This simplified SED model is needed in the absence of Spitzer data, where the DL07 models are insufficiently constrained. We also use this model in our subgalactic SED fits (Section 4.4) due to its computational simplicity.

The dust mass absorption coefficient at $\nu_{0}, \kappa$, is not well determined, and depends on the object investigated (see Netterfield et al. 2009 for a discussion). For each BLAST/ SINGS galaxy, we calculate a value for $\kappa$ by comparing the DL07 fit to the fit found for the spatially integrated modified blackbody SED (Section 4.3). The values of $\kappa$ for each galaxy are summarized in Table 3. These values are used in the calculation of the column density maps presented in Figure 9. For the galaxies without Spitzer observations, we use the mean value, $\kappa_{250}=0.29 \pm 0.03 \mathrm{~m}^{2} \mathrm{~kg}^{-1}$, to calculate dust mass from the modified blackbody fits. Assuming a $\kappa \propto v^{2}$ evolution, this corresponds to $k_{850}=0.025 \mathrm{~m}^{2} \mathrm{~kg}^{-1}$, smaller than the $k_{850}=0.07$ $\mathrm{m}^{2} \mathrm{~kg}^{-1}$ value calculated by James et al. (2002). It is also lower than the $250 \mu \mathrm{m}$ values of Draine \& Lee (1984) and Casey (1991).

For each fit, a brute-force grid search is performed to simultaneously fit $M_{\mathrm{d}}$ and $T$ to the data. The temperature, $T$, is sampled at 1000 uniformly spaced points between $5 \mathrm{~K}$ and $30 \mathrm{~K}$. This range was determined by first letting temperatures vary over a much wider range $(1-100 \mathrm{~K})$ and then discarding those portions of the parameter space discovered to be irrelevant to the fitting procedure. Because the dust mass, $M_{\mathrm{d}}$, may vary by several orders of magnitude, the sampling limits for the dust mass are automatically adjusted as necessary for the fit, and sampled uniformly at 1000 points. As with the DL07 models, goodness of fit is assessed using a $\chi^{2}$ test (Section 4.5 ). The marginalized likelihood is used to determine medians and $68 \%$ confidence intervals for the two fit parameters.

\subsection{Spatially Integrated SED Fits}

For the three galaxies without Spitzer data, we fit Equation (3) to BLAST plus IRAS $100 \mu \mathrm{m}$. The IRAS and BLAST measurements used to constrain the fits, and the results from these fits are presented in Figure 8, which also plots additional data from $I R A S, I S O$, and SCUBA, not used in this analysis.

For the four BLAST/SINGS galaxies, we also fit Equation (3) to BLAST, plus 160,100 , and $70 \mu \mathrm{m}$ data from MIPS or IRAS, to compare with the results of the DL07 models. These fits are plotted in Figure 8 as dashed curves. We note reasonably good agreement between the two models for all galaxies near the thermal peak. On the Wien side of the peak, as expected, the single-component modified blackbody quickly falls off in comparison to the DL07 model fit. On the Raleigh-Jeans side, due to the adoption of an emissivity index $\beta=2$, the modified blackbody also falls off faster than the DL07 model fit. For NGC 1097 and NGC 1291, the modified blackbody fit underpredicts the $500 \mu \mathrm{m}$ flux density by more than $1 \sigma$.

Dust temperatures from these fits for all galaxies in the sample are listed in Table 2, as are dust masses for the three galaxies without Spitzer observations.

\subsection{Subgalactic SED Fits}

Because the MIPS beams at $70 \mu \mathrm{m}$ and $160 \mu \mathrm{m}$ are of comparable size to the BLAST beams, we also investigate the dust properties on subgalactic scales for the four BLAST/ SINGS galaxies. To do this, the BLAST maps produced for the four galaxies were made on the same $9^{\prime \prime}$ pixel grid as the MIPS $160 \mu \mathrm{m}$ maps. The $70 \mu \mathrm{m}$ MIPS images, which have a compatibly aligned 4 ". 5 pixel grid, are rebinned to 9 " to put them on the same grid. All five maps are then convolved (smoothed) to the resolution of the $500 \mu \mathrm{m}$ data $\left(60^{\prime \prime}\right)$, and our modified blackbody template (Equation (3)) is fit to the data at each pixel in the map.

The central 12 pixels of NGC 1097 have $70 \mu \mathrm{m}$ surface brightness in excess of $200 \mathrm{MJy} \mathrm{sr}^{-1}$. For these 12 pixels, the $70 \mu \mathrm{m}$ data are excluded, due to possible nonlinearities, ${ }^{19}$ and the model is fit to four photometric points only.

Resultant temperature and column density maps for the four galaxies are presented in Figures 9 and 10. NGC 1510, the companion galaxy to NGC 1512 , appears quite prominently in these maps as seemingly hotter ( $>25 \mathrm{~K}$ ) than NGC 1512. This is not believed to be a real effect, and is likely due to poor performance by the fitting routine on the data for NGC 1510 , for which $\beta=2$ is probably not an appropriate choice.

Radially averaged temperature and column density profiles are plotted in Figure 11. We note a general decrease in both column density and dust temperature with increasing radius. Of the four galaxies, we notice two subgroups of two galaxies each. The brighter NGC 1097 and NGC 1566, for which BLAST detects high-intensity emission continuously from the core to disk of the galaxy, have relatively flat temperature profiles. NGC 1291 and NGC 1512, which BLAST detects with a more isolated core, fall off more steeply. We also potentially detect

\footnotetext{
19 http://data.spitzer.caltech.edu/popular/sings/20070410_enhanced_v1/ Documents/sings_fifth_delivery_v2.pdf
} 


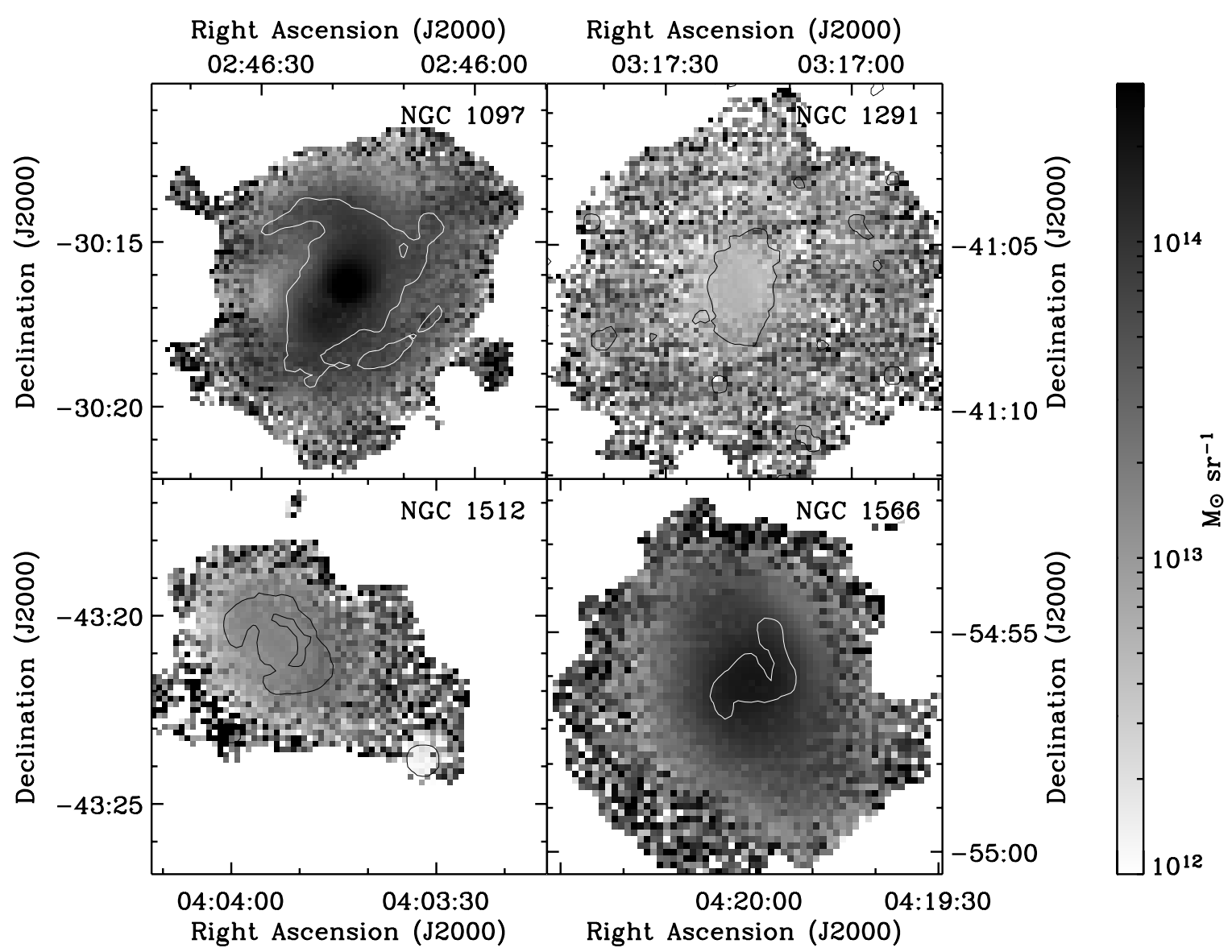

Figure 9. Dust column density maps based on BLAST and MIPS observations for the four BLAST/SINGS galaxies. Only those pixels whose $250 \mu \mathrm{m}$ flux density is inconsistent with zero at the $1 \sigma$ level are plotted. Contours plotted are the same as in Figure 9 . Mean dust column density errors in the high $\mathrm{S} / \mathrm{N}$ portions of the maps are $\pm 2.1 \times 10^{13} M_{\odot} \mathrm{sr}^{-1}, \pm 1.6 \times 10^{13} M_{\odot} \mathrm{sr}^{-1}, \pm 0.9 \times 10^{13} M_{\odot} \mathrm{sr}^{-1}$, and $\pm 2.1 \times 10^{13} M_{\odot} \mathrm{sr}^{-1}$ for NGC 1097, NGC 1291, NGC 1512, and NGC 1566, respectively.

the $1.5 \mathrm{kpc}$ star-forming ring in NGC 1097, which causes the kink in the temperature profile.

\section{5. $\chi^{2}$ Calculation}

We use $\chi^{2}$ minimization to assess goodness of fit for both the DL07 models and the modified blackbody model. The BLAST calibration uncertainties are highly correlated between observational bands (Truch et al. 2008, 2009). Given observational data, $s_{\mathrm{d}}$, and band-convolved model predictions, $\tilde{s}$, we compute

$$
\chi^{2} \equiv\left(\tilde{s}-s_{\mathrm{d}}\right)^{\mathrm{T}} C^{-1}\left(\tilde{s}-s_{\mathrm{d}}\right),
$$

where $C^{-1}$ is the inverse data covariance matrix and $(\cdot)^{\mathrm{T}}$ denotes the transpose. Given the calibration uncertainty correlation matrix, $\rho$, the data covariance matrix is

$$
C_{i j}=\delta_{i j}\left[\left(\sigma_{\mathrm{d}}^{2}\right)_{i}+\left(\sigma_{\mathrm{m}}^{2}\right)_{i}\right]+\rho_{i j}\left(\sigma_{\mathrm{c}}\right)_{i}\left(\sigma_{\mathrm{c}}\right)_{j},
$$

where $\sigma_{\mathrm{d}}$ is the measurement uncertainty, $\sigma_{\mathrm{c}}$ is the calibration uncertainty, and $\sigma_{\mathrm{m}}$ is an uncertainty associated with the model. For the DL07 models, in order to compare with their results, we follow Dr07 and adopt $\sigma_{\mathrm{m}}=0.1 s_{\mathrm{d}}$. For the modified blackbody model, we use $\sigma_{\mathrm{m}}=0$. We assume that IRAS and Spitzer uncertainties are uncorrelated between bands, and set $\rho_{i j}=\delta_{i j}$ for non-BLAST measurements.

For the BLAST measurements, we set $\rho$ to the value of the Pearson correlation matrices computed as outlined in Truch et al. $(2008,2009)$. For both the calibration uncertainty $\left(\sigma_{\mathrm{c}}\right)$ and the calibration uncertainty correlation matrix $(\rho)$, we add to the values given in those papers an additional source of uncertainty associated with our ability to measure the BLAST band passes. We estimate the error in the bandpass measurements to be at most $5 \%$. We additionally assume that this uncertainty is uncorrelated between bands.

The magnitude of the effect of bandpass measurement error on the BLAST calibration uncertainties depends on the spectrum of the object observed. A source with the same spectrum as our calibrators (e.g., for BLAST06, VY Canis Majoris, for which Truch et al. (2009) find a best-fit single-component modified blackbody with $T=346 \pm 19 \mathrm{~K}$ and $\beta=0.55 \pm 0.05$ ) is unaffected by bandpass errors, but for a source with a significantly different spectrum, the effect can be non-negligible, especially in the high $\mathrm{S} / \mathrm{N}$ case. This is the case in this study, and we have adjusted our calibration uncertainties accordingly.

Because the underlying spectrum of the galaxies is unknown, we use the modified blackbody model as a proxy. The calculated calibration uncertainties affect the best fit of the model, so this is an iterative process: we first fit the model using a guess for the calibration uncertainties, and then update the uncertainties based on the best fit. This process is iterated until convergence is achieved. In general, only one iteration after the initial guess is required for convergence.

For this procedure, we have calculated calibration uncertainties and uncertainty correlations for various $\beta=2$ modified 


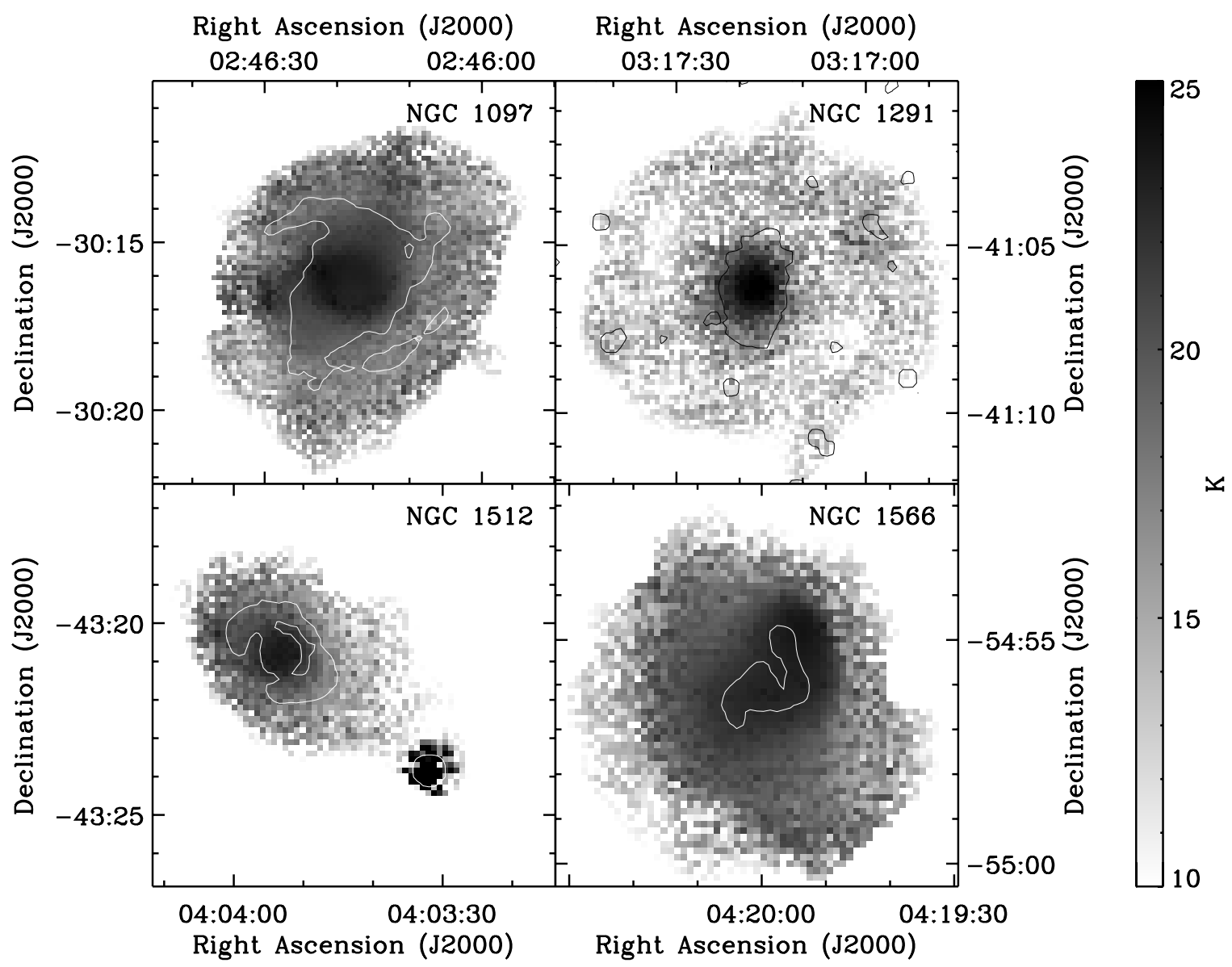

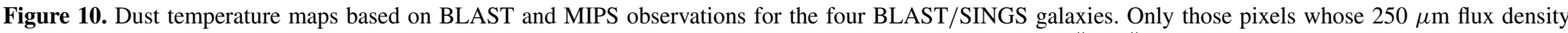

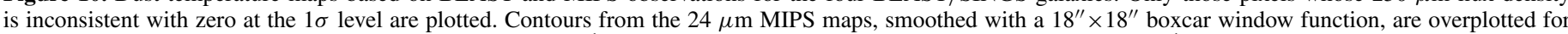

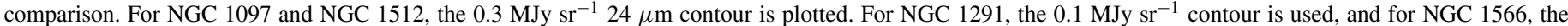

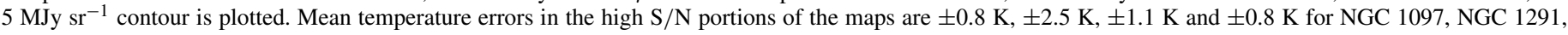
NGC 1512, and NGC 1566, respectively.

blackbody models with temperatures in the range $15-25 \mathrm{~K}$. The resulting calibration uncertainties vary most with the choice of model at $250 \mu \mathrm{m}(12 \%-14 \%$ for BLAST06), with smaller variations at $350 \mu \mathrm{m}(10.0 \%-10.5 \%)$ and $500 \mu \mathrm{m}(8.00 \%-8.25 \%)$. The uncertainty correlations calculated show similar behavior, varying between 0.80 and 0.97 for the $250-350 \mu \mathrm{m}$ correlation but only $0.91-0.92$ for the $350-500 \mu \mathrm{m}$ correlation.

When reporting goodness of fit, we report $\chi^{2}$ per degree of freedom:

$$
\chi_{r}^{2} \equiv \frac{1}{N_{\mathrm{b}}-N_{\mathrm{m}}} \chi^{2},
$$

where $N_{\mathrm{b}}$ is the number of observational bands considered in the fit, and $N_{\mathrm{m}}$ is the number of model parameters (five for the DL07 models and two for the modified blackbody model). The number of bands ranges from 4 (3 BLAST $+100 \mu \mathrm{m} I R A S)$ for modified blackbody fits in the absence of Spitzer data, to 13 (4 IRAC, 3 IRAS, 3 MIPS, 3 BLAST) when fitting the DL07 models.

\section{DISCUSSION}

\subsection{Dust Models}

Of the four galaxies observed by both BLAST and Spitzer, the DL07 fits we find for all but NGC 1291 are consistent with the best-fit models determined without BLAST data in Dr07. For NGC 1291, we find a dust mass approximately two times larger than Dr07. This discrepancy is due to the restriction $U_{\text {min }} \geqslant 0.7$ imposed by Dr07 in the absence of SCUBA data. We forego this restriction for our fit of NGC 1291, and find a best-fit $U_{\min }=0.2$. Constraining $U_{\min } \geqslant 0.7$ results in a fit consistent with that of $\operatorname{Dr} 07\left(q_{\mathrm{PAH}}=2.8 \%, U_{\min }=0.7, \log \left(M_{\mathrm{d}} / M_{\odot}\right)=\right.$ $7.36, \gamma=1.00 \%)$, but a poor fit to BLAST data $\left(\chi_{r}^{2}=2.59\right)$. This fit is plotted in Figure 8 for comparison.

The dust temperatures found in this study agree well with the 15-25 K temperatures found for the cold dust component in other studies of nearby galaxies (e.g., Alton et al. 1998; Braine \& Hughes 1999; Dunne \& Eales 2001; Xilouris et al. 2004; Vlahakis et al. 2005; Willmer et al. 2009).

\subsection{Fraction of Core to Total Emission}

BLAST's unique ability to spatially resolve the submillimeter flux of these nearby galaxies allows us to investigate the fraction of submillimeter flux which arises from the extended disks compared to their central core and circumnuclear regions. Although BLAST's resolution is insufficient to resolve the central AGN of these galaxies (where present), we can still put limits on the contribution made to the submillimeter flux by the nuclear accretion and star formation activity toward their circumnuclear regions (typically within a few kiloparsecs of the nucleus).

For each galaxy, except the edge-on NGC 4565, we find the $3 \mathrm{db}$ radial flux contour (that is, the radius at which the radially 
Table 4

Core Flux Density Fractions for BLAST Nearby Galaxies

\begin{tabular}{|c|c|c|c|c|c|}
\hline \multirow[t]{2}{*}{ Galaxy } & \multicolumn{2}{|c|}{$\begin{array}{c}\text { Core } \\
\text { Radius }\end{array}$} & \multirow{2}{*}{$\begin{array}{l}\text { Band } \\
(\mu \mathrm{m})\end{array}$} & \multirow{2}{*}{$\begin{array}{c}\text { Core } \\
\text { Flux } \\
(\mathrm{Jy})\end{array}$} & \multirow{2}{*}{$\begin{array}{c}\text { Core Flux } \\
\text { Fraction } \\
(\%)\end{array}$} \\
\hline & $\left({ }^{\prime}\right)$ & $(\mathrm{kpc})$ & & & \\
\hline \multirow[t]{3}{*}{ NGC 1097} & 0.37 & 1.8 & 250 & $13.35 \pm 0.03$ & $14.93 \pm 0.06$ \\
\hline & & & 350 & $4.97 \pm 0.02$ & $12.26 \pm 0.07$ \\
\hline & & & 500 & $1.36 \pm 0.01$ & $8.44 \pm 0.08$ \\
\hline \multirow[t]{3}{*}{ NGC 1291} & 0.71 & 2.0 & 250 & $1.41 \pm 0.02$ & $5.42 \pm 0.10$ \\
\hline & & & 350 & $0.64 \pm 0.01$ & $5.04 \pm 0.17$ \\
\hline & & & 500 & $0.23 \pm 0.01$ & $3.58 \pm 0.17$ \\
\hline \multirow[t]{3}{*}{ NGC 1365} & 0.29 & 1.6 & 250 & $13.97 \pm 0.16$ & $9.58 \pm 0.13$ \\
\hline & & & 350 & $5.38 \pm 0.10$ & $8.62 \pm 0.19$ \\
\hline & & & 500 & $1.35 \pm 0.05$ & $5.46 \pm 0.23$ \\
\hline \multirow[t]{3}{*}{ NGC 1512} & 0.40 & 1.2 & 250 & $1.51 \pm 0.01$ & $10.26 \pm 0.08$ \\
\hline & & & 350 & $0.61 \pm 0.01$ & $8.79 \pm 0.21$ \\
\hline & & & 500 & $0.14 \pm 0.01$ & $3.43 \pm 0.06$ \\
\hline \multirow[t]{3}{*}{ NGC 1566} & 0.98 & 5.1 & 250 & $19.07 \pm 0.04$ & $37.04 \pm 0.19$ \\
\hline & & & 350 & $7.72 \pm 0.04$ & $34.78 \pm 0.37$ \\
\hline & & & 500 & $2.84 \pm 0.02$ & $31.04 \pm 0.46$ \\
\hline \multirow[t]{3}{*}{ NGC 1808} & 0.29 & 0.9 & 250 & $10.86 \pm 0.01$ & $12.94 \pm 0.03$ \\
\hline & & & 350 & $3.66 \pm 0.01$ & $11.06 \pm 0.07$ \\
\hline & & & 500 & $0.92 \pm 0.01$ & $7.79 \pm 0.05$ \\
\hline
\end{tabular}

Note. Flux uncertainties reported are measurement errors $\left(\sigma_{\mathrm{d}}\right)$ only.

averaged flux has dropped to half of the peak flux) at $250 \mu \mathrm{m}$, and use this radius to define the core of the galaxy. Fluxes in all three BLAST bands from this core region are presented in Table 4. Also in this table is the "core fraction," the fraction of the total BLAST measured flux which originates in the core of the galaxy.

Most of the sample have core fractions in the range $7.5 \%-$ $15 \%$. NGC 1566 is a clear outlier, with roughly one third of the submillimeter flux in the core of the galaxy. The second brightest known Seyfert galaxy (de Vaucouleurs 1973), NGC 1566, has a core whose submillimeter flux originates largely from the AGN. The galaxy with the smallest core fraction is NGC 1291, a galaxy with no known active nucleus. Other than in NGC 1566, the bulk of the submillimeter emission in the core of the galaxy could be explained by nuclear star formation.

All galaxies show a decrease in core fraction with increasing wavelength, a result of the core dust temperature being generally warmer than the dust in the surrounding disk (see Figure 11). Other than NGC 1566, most galaxies have weak or no AGN, although BLAST is unable to separate AGN-derived flux from star formation in the central regions of the galaxies. In the case of NGC 1097, the unresolved $18^{\prime \prime}$ circumnuclear starburst ring (e.g., Hummel et al. 1987; Barth et al. 1995; Quillen et al. 1995; Kotilainen et al. 2000) likely provides a sizable contribution to the total core flux.

BLAST's ability to measure the compact to extended flux ratio of these galaxies is a result of BLAST's high sensitivity and resolution. This indicates that current and future, higher-sensitivity and higher-resolution FIR and submillimeter experiments, such as Herschel, SCUBA-2, SPICA, the James Webb Space Telescope (JWST), ALMA, Large Millimeter Telescope, and the Robert C. Byrd Green Bank Telescope, which will be able to spatially separate nonthermal components from extended star formation emission, are important for understanding the relationship between FIR-submillimeter luminosity and star formation rates in both local and high-redshift galaxies.

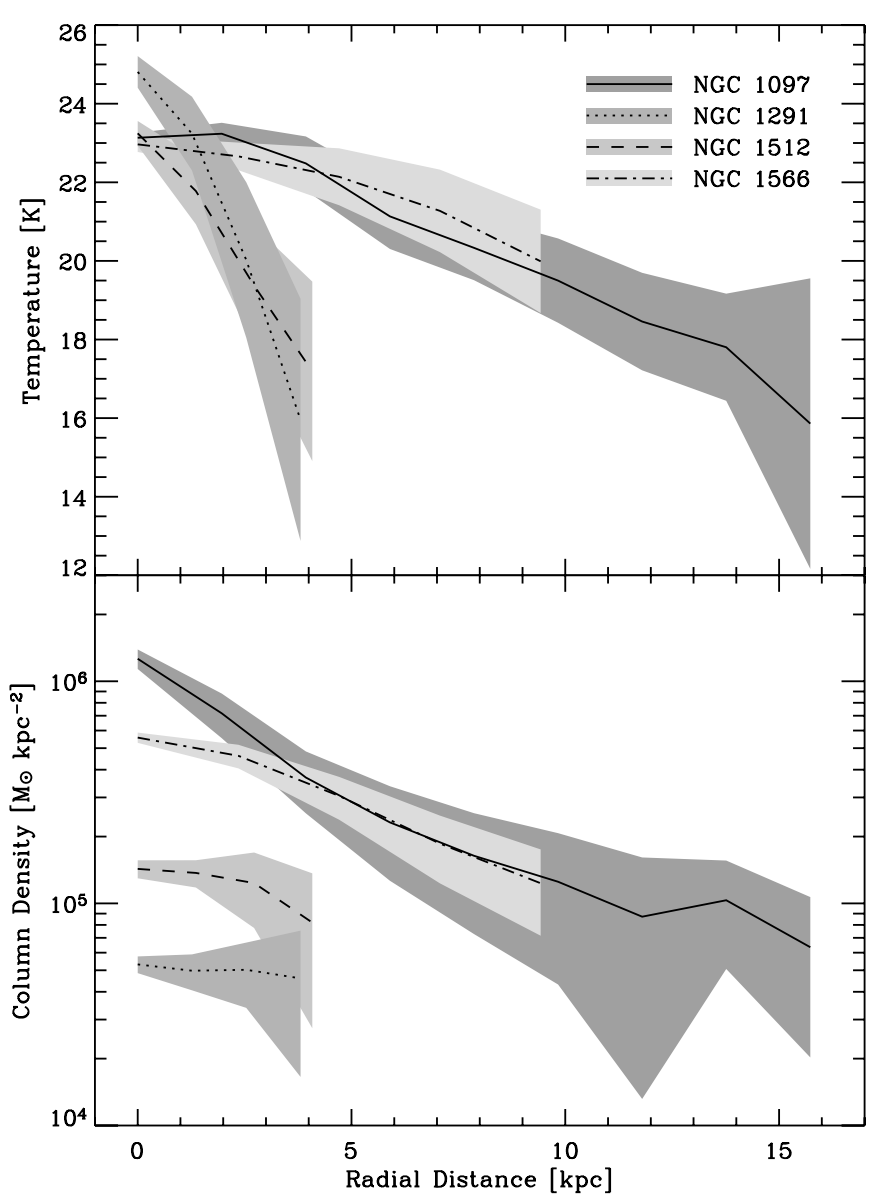

Figure 11. Mean radial dust temperature (top) and dust column density (bottom) for the four BLAST/SINGS galaxies, with $1 \sigma$ uncertainty bands. Resolution elements at $500 \mu \mathrm{m}$ are approximately 4.9, 5.2, 3.0, and 2.8 kpc for NGC 1097, NGC 1291, NGC 1512, and NGC 1566, respectively.

Table 5

Resolved Versus Unresolved Mass Estimates for BLAST Nearby Galaxies

\begin{tabular}{cccrc}
\hline \hline Galaxy & $\begin{array}{c}\text { Unresolved Mass }^{\mathrm{a}} \\
\log \left(\frac{M_{\mathrm{u}}}{M_{\odot}}\right)\end{array}$ & $\begin{array}{c}\text { Resolved Mass } \\
\log \left(\frac{M_{\mathrm{r}}}{M_{\odot}}\right)\end{array}$ & $\begin{array}{c}\text { Ratio } \\
\frac{M_{\mathrm{r}}}{M_{\mathrm{u}}}\end{array}$ & $\begin{array}{c}\text { Aperture } \\
\text { Diameter } \\
\left({ }^{\prime}\right)\end{array}$ \\
\hline NGC 1097 & 8.37 & $9.17_{-0.14}^{+0.13}$ & $6.3_{-1.7}^{+2.2}$ & 10.9 \\
NGC 1291 & 7.62 & $8.90_{-0.20}^{+0.17}$ & $19.1_{-7.0}^{+9.1}$ & 13.2 \\
NGC 1512 & 7.25 & $8.38_{-0.14}^{+0.12}$ & $13.5_{-3.7}^{+4.3}$ & 5.8 \\
NGC 1566 & 8.21 & $8.92_{-0.10}^{+0.09}$ & $5.1_{-1.1}^{+1.2}$ & 6.8 \\
\hline
\end{tabular}

Note. ${ }^{\text {a }}$ DL07 model mass estimate from Table 3.

\subsection{Comparison of Resolved and Unresolved Mass and Luminosity Estimates}

Our resolved measurements of the mass distribution in these galaxies allow us to investigate how mass estimates may be affected in more distant and smaller galaxies that are unresolved. For the four galaxies for which we have dust column density maps (Figure 9), we apply the same aperture used to calculate our total flux measurements to the column density maps. The masses contained in these apertures, along with the aperture diameters, are listed in Table 5.

In all cases, the resolved mass estimate is significantly higher than the mass estimate obtained by the DL07 fit to the unresolved total flux from the galaxy. The ratio of resolved to unresolved 
mass estimates ranges from $\sim 5$, for NGC 1566, to $\sim 19$, for NGC 1291. Furthermore, the mass ratio follows the behavior of the radial temperature profile (Figure 11), with a larger temperature gradient corresponding to a larger mass ratio. This effect is independent of the choice of $\kappa$, since both our resolved and unresolved mass estimates use the same $\kappa$ to derive dust mass from the SED fit.

As a check, we performed a similar analysis to measure the difference between resolved and unresolved luminosity estimates. Using the same aperture, we summed the 60-1000 $\mu \mathrm{m}$ luminosity in the resolved maps, and compared this to the 60$1000 \mu \mathrm{m}$ found from the modified blackbody fit to the galaxy as a whole. As expected, these values agree with one another to within measurement uncertainty.

Clearly, while luminosity, and hence star formation rate, is unaffected by resolution, accurate dust mass measurements rely on resolved observations. Since luminosity, and therefore observed flux density, is a strong function of temperature, there is a potential for unresolved observations of galaxies to hide cool dust. For the four galaxies we investigate, a disproportionate fraction of their total SEDs is produced by their relatively warmer, less massive cores. A single temperature modified blackbody fit to such data tends to infer a warmer temperature and shallower (i.e., smaller) value of $\beta$, and hence lower, incorrect, dust mass. Our data are consistent with the model of Dunne \& Eales (2001), who used a two-component $\beta=2$ modified blackbody SED to separate the cool (15-25 K) dust component from warmer $(T>30 \mathrm{~K})$ emission in the SCUBA Local Universe Galaxy Survey.

The upcoming higher-resolution experiments should be able to better estimate the quantity of dust in distant galaxies.

\section{SUMMARY}

BLAST made resolved observations of seven nearby galaxies over the course of two flights. Four of these galaxies, NGC 1097, NGC 1291, NGC 1512, and NGC 1566, have complementary Spitzer observations as part of the SINGS survey. For these four galaxies, we fit the models of Draine \& Li (2007) to BLAST, Spitzer, and IRAS observations. Best-fit parameters are tabulated in Table 3.

The best-fit models for three of these four galaxies are consistent with the best-fit models calculated in Draine et al. (2007) without BLAST data. For the fourth galaxy, NGC 1291, we find a dust mass roughly two times larger than the dust mass determined by Draine et al. (2007). We also calculate a value for the dust mass absorption coefficient at $250 \mu \mathrm{m}$ $\kappa=0.29 \pm 0.03 \mathrm{~m}^{2} \mathrm{~kg}^{-1}$ by comparing the Draine \& Li models with a modified blackbody model.

For these four galaxies, we also produce maps of dust column density and mean dust temperature based on BLAST and MIPS observations fit to a single-component modified blackbody model (Figures 9 and 10), as well as radial profiles of the same quantities (Figure 11). For the remaining three galaxies observed by BLAST but not observed by Spitzer, NGC 1365, NGC 1808, and NGC 4565, we calculate spatially integrated dust temperatures and dust masses by fitting a modified blackbody model to BLAST and IRAS data. We find mean dust temperatures for our galaxy sample to be in the range 16-23 K.

We calculate the fraction of the submillimeter detected flux originating in the core of the galaxy as a fraction of the total submillimeter emission from the galaxy. Although BLAST is unable to resolve the nucleus of these galaxies, the ratio of compact to extended flux measured by BLAST puts an important upper limit on the fraction of submillimeter radiation driven by a central AGN.

Only one of our samples, NGC 1365, the second brightest known Seyfert galaxy, has a significant fraction of its flux $(>30 \%)$ emanating from the core of the galaxy. In the remainder, the core fraction is small $(<15 \%)$ and may be accountable primarily to nuclear star formation. In all cases, the core fraction increases with decreasing wavelength, indicating that dust in the core of these galaxies is warmer than the dust in the surrounding disk, agreeing with the radial temperature profile of Figure 11.

We compare the total dust mass in our column density maps to the dust mass estimate based on the observation of the galaxies as a whole (i.e., an unresolved observation). We find 5-19 times more dust mass in the resolved maps than the unresolved estimate, a result of the unresolved measurements hiding cool dust. We perform a similar analysis with luminosity and find no discrepancy between the resolved and unresolved 60-1000 $\mu \mathrm{m}$ luminosity estimates.

Finally, for NGC 4565, we calculate a scale length of $118^{\prime \prime}$, $156^{\prime \prime}, 142^{\prime \prime}$ at $250 \mu \mathrm{m}, 350 \mu \mathrm{m}$, and $500 \mu \mathrm{m}$, respectively, in good agreement with measurements at other wavelengths.

The BLAST collaboration acknowledges the support of NASA through grant numbers NAG5-12785, NAG5-13301, and NNGO-6GI11G, the Canadian Space Agency (CSA), Canada's Natural Sciences and Engineering Research Council (NSERC), and the UK Particle Physics \& Astronomy Research Council (PPARC). We thank the Columbia Scientific Balloon Facility (CSBF) staff for their outstanding work. L.O. acknowledges partial support by the Puerto Rico Space Grant Consortium and by the Fondo Istitucional para la Investigacion of the University of Puerto Rico. C.B.N. acknowledges support from the Canadian Institute for Advanced Research. This research has been enabled by the use of WestGrid computing resources.

This work is based in part on observations made with the Spitzer Space Telescope, and has also made use of the NASA/IPAC Extragalactic Database (NED), both of which are operated by the Jet Propulsion Laboratory, California Institute of Technology, under contracts with the National Aeronautics and Space Administration. This research also made use of the SIMBAD database, operated at the Centre de Donées astronomiques de Strasbourg (CDS), Strasbourg, France.

\section{REFERENCES}

Alloin, D., Edmunds, M. G., Lindblad, P. O., \& Pagel, B. E. J. 1981, A\&A, 101, 377

Alton, P. B., et al. 1998, A\&A, 335, 807

Aumann, H. H., Fowler, J. W., \& Melnyk, M. 1990, AJ, 99, 1674

Barth, A. J., Ho, L. C., Filippenko, A. V., \& Sargent, W. L. 1995, AJ, 110 1009

Beck, R., Fletcher, A., Shukurov, A., Snodin, A., Sokoloff, D. D., Ehle, M., Moss, D., \& Shoutenkov, V. 2005, A\&A, 444, 739

Boselli, A., et al. 2008, in SF2A-2008, ed. C. Charbonnel, F. Combes, \& R. Samadi (Les Ulis: EDP Sciences), 361

Bottema, R. 1992, A\&A, 257, 69

Braine, J., \& Hughes, D. H. 1999, A\&A, 344, 779

Bregman, J. N., Hogg, D. E., \& Roberts, M. S. 1995, ApJ, 441, 561

Casey, S. C. 1991, ApJ, 371, 183

Chapin, E. L., et al. 2008, ApJ, 681, 428

Dale, D. A., et al. 2005, ApJ, 633, 857

Dale, D. A., et al. 2007, ApJ, 655, 863

de Vaucouleurs, G. 1973, ApJ, 181, 31

de Vaucouleurs, G. 1975, ApJS, 29, 193

de Vaucouleurs, G., de Vaucouleurs, A., Corwin, H. G., Jr., Buta, R. J., Paturel, G., \& Fouque, P. 1991, Third Reference Catalogue of Bright Galaxies (Berlin: Springer) 
Devlin, M. J., et al. 2009, Nature, 458, 737

Draine, B. T., \& Lee, H. M. 1984, ApJ, 285, 89

Draine, B. T., \& Li, A. 2007, ApJ, 657, 810

Draine, B. T., et al. 2007, ApJ, 663, 866

Dunne, L., \& Eales, S. A. 2001, MNRAS, 327, 697

Eales, S., Lilly, S., Webb, T., Dunne, L., Gear, W., Clements, D., \& Yun, M. 2000, AJ, 120, 2244

Engargiola, G., \& Harper, D. A. 1992, ApJ, 394, 104

Fazio, G. G., et al. 2004, ApJS, 154, 10

Fixsen, D. J., Dwek, E., Mather, J. C., Bennett, C. L., \& Shafer, R. A. 1998, ApJ, 508,123

Förster Schreiber, N. M., Sauvage, M., Charmandaris, V., Laurent, O., Gallais, P., Mirabel, I. F., \& Vigroux, L. 2003, A\&A, 399, 833

Galliano, E., Alloin, D., Pantin, E., Lagage, P. O., \& Marco, O. 2005, A\&A, 438,803

Gerin, M., Combes, F., \& Nakai, N. 1988, A\&A, 203, 44

Griffin, M., et al. 2007, Adv. Space Res., 40, 612

Hogg, D. E., Roberts, M. S., Bregman, J. N., \& Haynes, M. P. 2001, AJ, 121, 1336

Holland, W. S., et al. 1999, MNRAS, 303, 659

Hsieh, P.-Y., Matsushita, S., Lim, J., Kohno, K., \& Sawada-Satoh, S. 2008, ApJ, 683,70

Hughes, D. H., et al. 1998, Nature, 394, 241

Hummel, E., van der Hulst, J. M., \& Keel, W. C. 1987, A\&A, 172, 32

James, A., Dunne, L., Eales, S., \& Edmunds, M. G. 2002, MNRAS, 335, 753

Jiménez-Bailón, E., Santos-Lleó, M., Dahlem, M., Ehle, M., Mas-Hesse, J. M., Guainazzi, M., Heckman, T. M., \& Weaver, K. A. 2005, A\&A, 442, 861

Junkes, N., Zinnecker, H., Hensler, G., Dahlem, M., \& Pietsch, W. 1995, A\&A, 294, 8

Kennicutt, R. C., Jr., et al. 2003, PASP, 115, 928

Kinman, T. D. 1978, AJ, 83, 764

Komossa, S., \& Schulz, H. 1998, A\&A, 339, 345

Kotilainen, J. K., Reunanen, J., Laine, S., \& Ryder, S. D. 2000, A\&A, 353, 834

Lawrence, A., et al. 1999, MNRAS, 308, 897

Lorre, J. J. 1978, ApJ, 222, L99

Maiolino, R., et al. 2003, MNRAS, 344, L59

Maoz, D., Barth, A. J., Ho, L. C., Sternberg, A., \& Filippenko, A. V. 2000, BAAS, 32, 1529
Mason, R. E., Levenson, N. A., Packham, C., Elitzur, M., Radomski, J., Petric, A. O., \& Wright, G. S. 2007, ApJ, 659, 241

Mathis, J. S., Mezger, P. G., \& Panagia, N. 1983, A\&A, 128, 212

Mortier, A. M. J., et al. 2005, MNRAS, 363, 563

Neininger, N., Guelin, M., Garcia-Burillo, S., Zylka, R., \& Wielebinski, R. 1996, A\&A, 310, 725

Netterfield, C. B., et al. 2009, ApJ, 707, 1824

Nutter, D., \& Ward-Thompson, D. 2007, MNRAS, 374, 1413

Pascale, E., et al. 2008, ApJ, 681, 400

Patanchon, G., et al. 2008, ApJ, 681, 708

Pérez, I., \& Freeman, K. 2006, A\&A, 454, 165

Prunet, S., Netterfield, C. B., Hivon, E., \& Crill, B. P. 2000, in Proc. 35th Rencontre de Moriond, Energy Densities in the Universe, ed. J. Trân Thanh Vân (Gif-sur-Yvette: Editions Frontiéres)

Puget, J.-L., Abergel, A., Bernard, J.-P., Boulanger, F., Burton, W. B., Desert, F.-X., \& Hartmann, D. 1996, A\&A, 308, L5

Quillen, A. C., Frogel, J. A., Kuchinski, L. E., \& Terndrup, D. M. 1995, AJ, 110,156

Rice, W., Merrill, K. M., Gatley, I., \& Gillett, F. C. 1996, AJ, 112, 114

Rieke, G. H., et al. 2004, ApJS, 154, 25

Scott, S. E., et al. 2002, MNRAS, 331, 817

Smail, I., Ivison, R. J., \& Blain, A. W. 1997, ApJ, 490, L5

Soifer, B. T., \& Neugebauer, G. 1991, AJ, 101, 354

Spinoglio, L., Andreani, P., \& Malkan, M. A. 2002, ApJ, 572, 105

Stevens, J. A., Amure, M., \& Gear, W. K. 2005, MNRAS, 357, 361

Stickel, M., Lemke, D., Klaas, U., Krause, O., \& Egner, S. 2004, A\&A, 422, 39

Truch, M. D. P., et al. 2009, ApJ, 707, 1723

Truch, M. D. P., et al. 2008, ApJ, 681, 415

van der Kruit, P. C., \& Searle, L. 1981, A\&A, 95, 105

Véron, P., Lindblad, P. O., Zuiderwijk, E. J., Veron, M. P., \& Adam, G. 1980 A\&A, 87, 245

Vlahakis, C., Dunne, L., \& Eales, S. 2005, MNRAS, 364, 1253

Weingartner, J. C., \& Draine, B. T. 2001, ApJ, 548, 296

Wiebe, D. V. 2008, PhD thesis, Univ. Toronto

Willmer, C. N. A., Rieke, G. H., LeFloc'h, E., Hinz, J. L., Engelbracht, C. W., Marcillac, D., \& Gordon, K. D. 2009, AJ, 138, 146

Wilson, C. D., et al. 2009, ApJ, 693, 1736

Wolstencroft, R. D., \& Zealey, W. J. 1975, MNRAS, 173, 51P

Xilouris, E. M., Georgakakis, A. E., Misiriotis, A., \& Charmandaris, V. 2004, MNRAS, 355, 57 